Research Article

\title{
SINS/BDS Integrated Navigation for Hypersonic Boost-Glide Vehicles in the Launch-Centered Inertial Frame
}

\author{
Kai Chen $\mathbb{D}^{1},{ }^{1}$ Fuqiang Shen $\mathbb{D}^{1},{ }^{1}$ Jun Zhou $\mathbb{D},{ }^{1}$ and Xiaofeng $\mathrm{Wu} \mathbb{D}^{2}$ \\ ${ }^{1}$ Northwestern Polytechnical University, Xi'an 710072, Shaanxi, China \\ ${ }^{2}$ School of Aerospace, Mechanical and Mechatronic Engineering, University of Sydney, Sydney, Australia \\ Correspondence should be addressed to Kai Chen; chenkai@nwpu.edu.cn
}

Received 14 August 2020; Revised 18 October 2020; Accepted 27 October 2020; Published 16 November 2020

Academic Editor: Yongmin Zhong

Copyright (c) 2020 Kai Chen et al. This is an open access article distributed under the Creative Commons Attribution License, which permits unrestricted use, distribution, and reproduction in any medium, provided the original work is properly cited.

According to the trajectory specialty of hypersonic boost-glide vehicles, a strapdown inertial navigation system/BeiDou navigation satellite system (SINS/BDS) algorithm based on the launch-centered inertial (LCI) frame for hypersonic vehicles is proposed. First, the related frame system, especially the launch earth-centered inertial (LECI) frame, and the SINS mechanization in the LCI frame are introduced. Second, SINS discrete updating algorithms in the LCI frame for the compensation of coning, sculling, and scrolling effects are deduced in the attitude, velocity, and position updating algorithms, respectively. Subsequently, the Kalman filter of the SINS/BDS integrated navigation in the LCI frame is obtained. The method of converting BDS receiver position and velocity from the Earth-centered Earth-fixed (ECEF) frame to the LCI frame is deduced through the LECI frame. Finally, taking the typical hypersonic boost-glide vehicles as the object, the SINS/BDS algorithm vehicle field test and hardware-in-the-loop simulation are performed.

\section{Introduction}

A hypersonic vehicle is an aircraft that has the ability to fly and cruise for a long time at more than five times the speed of sound in near space; it is also called a "near-space hypersonic vehicle" [1]. The near space is the atmospheric space $20-100 \mathrm{~km}$ from the ground, and it is located below the low-orbit satellite operation area and above the traditional aircraft flight area. It has great military and civilian application value $[2,3]$.

Scholars from all over the world have studied many different navigation frames for different hypersonic vehicle navigation algorithms [4]. Stephen studied the strapdown algorithm in the earth-centered inertial frame for the SHEFEX2 hypersonic vehicle [5]. Mu et al. studied an integrated navigation algorithm of hypersonic aircraft in the ECI frame [6]. Wei et al. studied a hypersonic vehicle GFSINS/GPS/CNS algorithm using the ECEF frame [7]. Yu et al. used the NEU geography coordinate system as the hypersonic vehicle navigation reference system [8]. The $\mathrm{X}-43 \mathrm{~A}$ used an INS/GPS integrated navigation system and the geography coordinate system navigation algorithm [9]. Kang et al. studied an INS/BDS integrated navigation method in the ENU geography coordinate system [10]. Li studied the aerial alignment method of the integrated navigation system in the local horizontal coordinate system [11].

Because a hypersonic boost-glide vehicle is launched vertically, its trajectory is similar to that of the launch vehicle. If the local horizontal frame is used as the navigation coordinate system of the hypersonic boost-glide vehicle, the attitude angle will be singular during the navigation calculation process. Therefore, the LCI frame is often used as the navigation coordinate system of the launch vehicle, which will not cause the attitude angle to be singular, making the LCI frame more advantageous over the local horizontal frame $[4,12,13]$. According to the navigation solutions in the LCI frame, scholars from all over the world have designed some hypersonic vehicle flight control algorithms. Zhang et al. studied stellar-inertial integrated guidance to responsive launch vehicles in an LCI frame [12]. Zhao et al. built an INS/CNS/GPS system in the LCI frame for vehicles 
for reducing the effects of an imprecise Earth model and completing long-duration maneuvers within a wide flight envelope [13]. Our group proposed an algorithm of SINS in the LCI frame for hypersonic boost-glide vehicles and presented a solution in the local horizontal frame from the LCI frame.

In the near-space hypersonic flight, factors such as aerodynamic disturbance, scramjet operation, and steering gear operation affect the accuracy of the SINS of hypersonic vehicles. The Chinese BDS navigation system has become a world-class global navigation satellite system (GNSS) $[14,15]$. The application of BDS is less in hypersonic vehicles. In the present study, based on the high-dynamic characteristics of hypersonic vehicles, we propose updating algorithms for the attitude, velocity, and position of strapdown inertial navigation as well as a SINS/BDS integrated navigation system in the LCI frame. In addition, we conduct a test on the SINS/BDS integrated navigation vehicle and hardware-in-the-loop (HWIL) simulation of the LCI frame.

\section{SINS Mechanization in Launch-Centered Inertial Frame}

2.1. Common Frame Systems. In the SINS, the commonly used frame systems include the Earth-centered inertial frame (ECI, $i$ frame), Earth-centered Earth-fixed frame (ECEF, $e$ frame), launch Earth-centered inertial frame (LECI, $t$ frame), launch-centered Earth-fixed frame (LCEF, $g$ frame), launchcentered inertial frame (LCI, $a$ frame), and body-fixed frame (BF, $b$ frame) [4]. This study uses the frame systems described below and the LCI frame as the navigation frame system.

\subsection{Relationship between Frame Systems}

2.2.1. LCI Frame and BF Frame. The direction cosine matrix (DCM) from the LCI frame to the BF frame is $\mathbf{R}_{a}^{b}$, as shown in

$$
\mathbf{R}_{a}^{b}=\mathbf{R}_{x}(\gamma) \mathbf{R}_{y}(\psi) \mathbf{R}_{z}(\varphi)
$$

where $\varphi, \psi$, and $\gamma$ are the vehicle pitch, yaw, and roll angles in the LCI frame, respectively. $\mathbf{R}_{i}(\theta)$ is the DCM rotating around the $i$-axis $(i=x, y, z)$ by angle $\theta$.

2.2.2. LCEF Frame and ECEF Frame. The DCM from the ECEF frame to the LECF frame is $\mathbf{R}_{e}^{g}$, as shown in

$$
\mathbf{R}_{e}^{g}=\mathbf{R}_{y}\left(-90^{\circ}-\alpha_{0}\right) \mathbf{R}_{x}\left(\phi_{0}\right) \mathbf{R}_{z}\left(\lambda_{0}-90^{\circ}\right),
$$

where $\phi_{0}, \lambda_{0}$, and $\alpha_{0}$ are the initial latitude, initial longitude, and launch azimuth of the vehicle. In addition, at the time of launch, the coordinates of the LCEF, and LCI frames are in the same direction, and the coordinates of the LECI and ECEF frames are in the same direction. Therefore, the DCMs $\mathbf{R}_{t}^{a}$ from the LECI frame to the LCI frame and $\mathbf{R}_{e}^{g}$ are the same. During flight, $\mathbf{R}_{t}^{a}$ and $\mathbf{R}_{e}^{g}$ are constant values.

\subsection{Initial Value of Launch Time}

2.3.1. Initial Values of the Launch Point. The launch point position in the ECEF frame is $\mathbf{P}_{0}^{e}=\left[x_{e 0}, y_{e 0}, z_{e 0}\right]^{T}$, as given by

$$
\left\{\begin{array}{l}
x_{e 0}=\left(N_{0}+H_{0}\right) \cos \phi_{0} \cos \lambda_{0}, \\
y_{e 0}=\left(N_{0}+H_{0}\right) \cos \phi_{0} \sin \lambda_{0}, \\
z_{e 0}=\left[N_{0}\left(1-e^{2}\right)+H_{0}\right] \sin \phi_{0},
\end{array}\right.
$$

where $H_{0}$ is the initial height, $N_{0}=a_{e} / \sqrt{1-e^{2} \sin ^{2} L_{0}}, e$ is the eccentricity, and $a_{e}$ is the semimajor axis.

At the time of the launch, the coordinates of the LECI and ECEF frames point in the same direction, so $\mathbf{P}_{0}^{t}=\mathbf{P}_{0}^{e}$.

2.3.2. Geocentric Vector of the Launch Point. The geocentric vector of the launch point in the LCEF frame is $\mathbf{P}_{0 e}^{g}$ :

$$
\mathbf{P}_{e 0}^{g}=\mathbf{R}_{e}^{g} \mathbf{P}_{0}^{e}
$$

At the time of launch, the coordinates of the LCEF and LCI frames point in the same direction, so $\mathbf{P}_{0 e}^{a}=\mathbf{P}_{0 e}^{g}$.

2.3.3. Rotational Angular Velocity in the LCI Frame. The Earth's rotation angular velocity in the LECF frame relative to the LCI frame is $\omega_{\mathrm{ag}}^{\mathrm{g}}$.

$$
\omega_{\mathrm{ag}}^{g}=\omega_{\mathrm{ie}}\left[\begin{array}{c}
\cos \phi_{0} \cos \alpha_{0} \\
\sin \phi_{0} \\
-\cos \phi_{0} \sin \alpha_{0}
\end{array}\right]=\omega_{\mathrm{ie}} \omega_{e}^{0} .
$$

2.4. SINS Mechanization in the LCI Frame. The navigation equation of the SINS in the LCI frame is given by the following equation, and its mechanization is shown in Figure 1:

$$
\left[\begin{array}{c}
\dot{P}^{a} \\
\dot{V}^{a} \\
\dot{R}_{b}^{a}
\end{array}\right]=\left[\begin{array}{c}
\mathbf{V}^{a} \\
\mathbf{R}_{b}^{a} \mathbf{f}^{b}+\mathbf{g}^{a} \\
\mathbf{R}_{b}^{a} \mathbf{\Omega}_{\mathrm{ab}}^{b}
\end{array}\right] .
$$

As shown in (6) and Figure 1, the SINS algorithm is executed by the navigation computer, and steps involved are as follows. First, the vehicle attitude is obtained by integrating angular velocity $\omega^{b}$ measured by gyroscopes, and then attitude matrix $\mathbf{R}_{b}^{a}$ is obtained. Second, specific force $\mathbf{f}^{b}$ measured by accelerometers is converted to the LCI frame through attitude matrix $\mathbf{R}_{b}^{a}$; considering the effect of gravity $\mathbf{g}^{a}$, velocity $\mathbf{V}^{a}$ of the vehicle is obtained by integrating the sum of $\mathbf{f}^{a}$ and $\mathbf{g}^{a}$ in the LCI frame. Finally, the position $\mathbf{P}^{a}$ of the vehicle is obtained by integrating velocity $\mathbf{V}^{a}$, and $\mathbf{p}^{g}=[x, y, z]^{T}$. These three integral processes are termed SINS attitude updating, velocity updating, and position updating, respectively.

The formula for calculating gravity in the LCI frame is found in reference:

$$
\mathbf{g}^{a}=g_{r}^{\prime} \mathbf{r}^{0}+g_{\omega_{e}} \boldsymbol{\omega}_{e}^{0}
$$




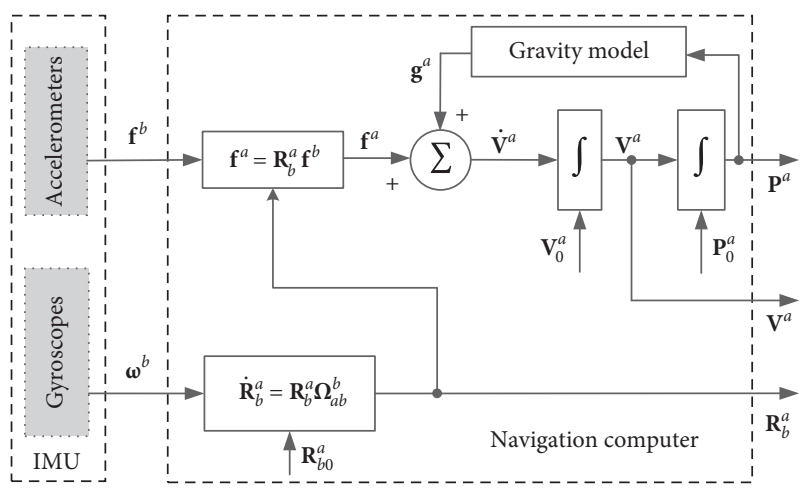

FIGURE 1: Mechanization of SINS in the LCI frame.

where $g_{r}^{\prime}$ is calculated by (8) and $g_{\omega_{e}}$ is calculated by (9). $r^{0}$ is the unit vector of $\mathbf{r}$, and $\mathbf{r}$ is calculated by (10):

$$
\begin{aligned}
g_{r}^{\prime} & =-\frac{\mu}{r^{2}}\left[1+J\left(\frac{a_{e}}{r}\right)^{2}\left(1-5 \sin ^{2} \phi\right)\right], \\
g_{\omega_{e}} & =-2 \frac{\mu}{r^{2}} J\left(\frac{a_{e}}{r}\right)^{2} \sin \phi, \\
\mathbf{r} & =\mathbf{P}_{0 e}^{a}+\mathbf{P}^{a} .
\end{aligned}
$$

In these equations, $\mu$ is the coefficient of gravity; $J=(3 / 2) J_{2}$, where $J_{2}$ is the harmonic coefficient; $r=\sqrt{x^{2}+y^{2}+z^{2}} ; \phi$ is the Earth-centered latitude, $\phi=\arcsin \left(r w /\left(|r| * \omega_{\text {ie }}\right)\right)$; and $r w=\mathbf{r} \cdot \omega_{e}$.

The initial value of position $\mathbf{P}^{a}$ is $\mathbf{P}_{0}^{a}=[0,0,0]^{T}$, and the initial value of attitude matrix $\mathbf{R}_{a}^{b}$ is determined by the initial attitude angles $\varphi_{0}, \psi_{0}$, and $\gamma_{0}$ in the initial alignment. The initial value $\mathbf{V}_{0}^{a}$ is obtained as follows:

$$
\mathbf{V}_{0}^{a}=\boldsymbol{\omega}_{\mathrm{ag}}^{g} \times \mathbf{P}_{0 e}^{g} .
$$

\section{SINS Numerical Updating Algorithm in the LCI Frame}

The hypersonic flight is generally affected by coning, sculling, and scrolling effects $[16,17]$. Therefore, the compensation algorithms for the three effects are derived in attitude updating [18], velocity updating [19], and position updating [19], respectively.

In this study, the two-sample updating algorithm is used; in other words, the angular velocity vector $\omega^{b}$ from the gyroscope and the specific force measurement vector $\mathbf{f}^{b}$ from the accelerometer are both assumed to be linear models; that is,

$$
\left\{\begin{array}{l}
\boldsymbol{\omega}^{b}(t)=\mathbf{a}+2 \mathbf{b}\left(t-t_{k-1}\right), \\
\mathbf{f}^{b}(t)=\mathbf{A}+2 \mathbf{B}\left(t-t_{k-1}\right),
\end{array}\right.
$$

where $\mathbf{a}, \mathbf{b}, \mathbf{A}, \mathbf{B}$ are constant vectors; then, we can get the angular and velocity increment expressions,

$$
\left\{\begin{array}{l}
\Delta \boldsymbol{\theta}_{k}=\boldsymbol{\theta}^{b}\left(t, t_{k-1}\right)=\int_{t_{k-1}}^{t} \boldsymbol{\omega}^{b}(\tau) \mathrm{d} \tau=\mathbf{a}\left(t-t_{k-1}\right)+\mathbf{b}\left(t-t_{k-1}\right)^{2}, \\
\Delta \mathbf{V}_{k}=\mathbf{V}^{b}\left(t, t_{k-1}\right)=\int_{t_{k-1}}^{t} \mathbf{f}^{b}(\tau) \mathrm{d} \tau=\mathbf{A}\left(t-t_{k-1}\right)+\mathbf{B}\left(t-t_{k-1}\right)^{2} .
\end{array}\right.
$$

If the IMU is sampled twice at equal intervals in $\left[t_{k-1}, t_{k}\right]$, the sampling time is $t_{k-1 / 2}$ and $t_{k}$, respectively, $T=t_{k}-t_{k-1}$ and $h=T / 2$ are recorded; then we can get

$$
\left\{\begin{array}{l}
\Delta \boldsymbol{\theta}_{1}=\int_{t_{k-1}}^{t_{k-1}+h} \boldsymbol{\omega}^{b}(\tau) \mathrm{d} \tau=h \mathbf{a}+h^{2} \mathbf{b}=\frac{1}{2} \mathbf{a} T+\frac{1}{4} \mathbf{b} T^{2}, \\
\Delta \boldsymbol{\theta}_{2}=\int_{t_{k-1}+h}^{t_{k}} \boldsymbol{\omega}^{b}(\tau) \mathrm{d} \tau=h \mathbf{a}+3 h^{2} \mathbf{b}=\frac{1}{2} \mathbf{a} T+\frac{3}{4} \mathbf{b} T^{2}, \\
\Delta \mathbf{V}_{1}=\int_{t_{k-1}}^{t_{k-1}+h} \mathbf{f}^{b}(\tau) \mathrm{d} \tau=h \mathbf{A}+h^{2} \mathbf{B}, \\
\Delta \mathbf{V}_{2}=\int_{t_{k-1}+h}^{t_{k}} \mathbf{f}^{b}(\tau) \mathrm{d} \tau=h \mathbf{A}+3 h^{2} \mathbf{B},
\end{array}\right.
$$

where $\Delta \theta_{1}$ and $\Delta \mathbf{V}_{1}$ are the angular and velocity increments in the time period $\left[t_{k-1}, t_{k-1}+h\right]$, and $\Delta \theta_{2}$ and $\Delta \mathbf{V}_{2}$ are the angular and velocity increments in the time period $\left[t_{k-1}+h, t_{k}\right]$. Meanwhile, $\Delta \theta_{k}=\Delta \theta_{1}+\Delta \theta_{2} \quad$ and $\Delta \mathbf{V}_{k}=\Delta \mathbf{V}_{1}+\Delta \mathbf{V}_{2}$.

3.1. Attitude Updating Algorithm. The quaternion attitude updating algorithm based on rotation vector is completed in two steps: (1) calculation of a rotation vector, which describes the state change of the vehicle attitude, and (2) update of quaternions.

The recurrence formula of attitude quaternion updating is as follows:

$$
\mathbf{q}_{b(k)}^{a}=\mathbf{q}_{b(k-1)}^{a} \mathbf{q}_{b(k)}^{b(k-1)},
$$

where the time before and after attitude updating is $t_{k-1}$ and $t_{k}$, during which the quaternions of attitude are $\mathbf{q}_{b(k-1)}^{a}$ and $\mathbf{q}_{b(k)}^{a}$, respectively. Quaternion $\mathbf{q}_{b(k)}^{b(k-1)}$ is calculated by angular velocity $\omega_{a b}^{b}(t)$, which is angular velocity $\omega^{b}$.

Note that $\Phi_{k}$ is the equivalent rotation vector of $\mathrm{BF}$ frame relative to the LCI frame, and the corresponding equivalent vector differential equation approximated as follows:

$$
\dot{\Phi}_{k} \approx \boldsymbol{\omega}_{\mathrm{ab}}^{b}+\frac{1}{2} \boldsymbol{\Phi}_{k} \times \boldsymbol{\omega}_{\mathrm{ab}}^{b}+\frac{1}{12} \boldsymbol{\Phi}_{k} \times\left(\boldsymbol{\Phi}_{k} \times \boldsymbol{\omega}_{\mathrm{ab}}^{b}\right) \approx \boldsymbol{\omega}_{\mathrm{ab}}^{b}+\frac{1}{2} \boldsymbol{\Phi}_{k} \times \boldsymbol{\omega}_{\mathrm{ab}}^{b} .
$$

Since the output of the optical gyroscope is an angle increment, it is necessary to solve the rotation vector by the Taylor series expansion method. Take $\Phi_{k}$ as the equivalent rotation vector in the time period $\left[t_{k-1}, t_{k}\right]$; then, the Taylor series expansion of $\Phi_{k}(T)$ is shown in 
$\Phi_{k}(T)=\Phi_{k-1}(0)+T \dot{\Phi}_{k-1}(0)+\frac{T^{2}}{2 !} \ddot{\Phi}_{k-1}(0)+\frac{T^{3} \dot{t}}{3 !} \Phi_{k-1}(0)+\cdots$
From (12), the derivative of each order of the vehicle angular velocity is obtained as follows:

$$
\left\{\begin{array}{l}
\boldsymbol{\omega}_{a b}^{b}\left(t_{k-1}\right)=\left.\boldsymbol{\omega}_{a b}^{b}\left(t_{k-1}+\tau\right)\right|_{\tau=0}=\mathbf{a}, \\
\dot{\omega}_{a b}^{b}\left(t_{k-1}\right)=\left.\dot{\omega}_{a b}^{b}\left(t_{k-1}+\tau\right)\right|_{\tau=0}=2 \mathbf{b}, \\
\boldsymbol{\omega}_{a b}^{b(i)}\left(t_{k-1}\right)=\left.\boldsymbol{\omega}_{a b}^{b(i)}\left(t_{k-1}+\tau\right)\right|_{\tau=0}=0, \quad i=2,3,4, \ldots
\end{array}\right.
$$

From (13), the derivative of each order of the angular increment is obtained as follows:

$$
\left\{\begin{array}{l}
\Delta \boldsymbol{\theta}(0)=\left.\Delta \boldsymbol{\theta}(\tau)\right|_{\tau=0}=\mathbf{a} \tau+\mathbf{b} \tau^{2}=0 \\
\Delta \dot{\theta}(0)=\left.\Delta \dot{\theta}(\tau)\right|_{\tau=0}=\left.\boldsymbol{\omega}_{a b}^{b}\left(t_{k}+\tau\right)\right|_{\tau=0}=\mathbf{a}, \\
\Delta \ddot{\theta}(0)=\left.\Delta \ddot{\theta}(\tau)\right|_{\tau=0}=\left.\dot{\omega}_{a b}^{b}\left(t_{k}+\tau\right)\right|_{\tau=0}=2 \mathbf{b}, \\
\Delta \boldsymbol{\theta}^{(i)}(0)=\left.\Delta \boldsymbol{\theta}^{(i)}(\tau)\right|_{\tau=0}=\left.\boldsymbol{\omega}_{a b}^{b(i-1)}\left(t_{k}+\tau\right)\right|_{\tau=0}=0, \quad i=3,4,5, \ldots
\end{array}\right.
$$

Further, because the attitude updating period $T$ is generally in milliseconds, $\Phi_{k}$ can also be regarded as a small magnitude. Therefore, when calculating the derivative of $\Phi_{k}(\tau)$ at $\tau=0$, according to (16), replace the second term of $\Phi_{k}(\tau)$ with the angle increment; then, we can get

$$
\Phi_{k}(\tau) \approx \Delta \boldsymbol{\theta}(\tau)
$$

Thus, we can get the following equation from (16):

$$
\dot{\Phi}_{k}(\tau)=\boldsymbol{\omega}_{\mathrm{ab}}^{b}\left(t_{k-1}+\tau\right)+\frac{1}{2} \Delta \boldsymbol{\theta}(\tau) \times \boldsymbol{\omega}_{\mathrm{ab}}^{b}\left(t_{k-1}+\tau\right), \quad 0 \leq \tau \leq T .
$$

Considering (18) and (19), the derivative of each order of (21) is obtained:

$$
\left\{\begin{array}{l}
\ddot{\Phi}_{k}(\tau)=\dot{\omega}_{\mathrm{ab}}^{b}\left(t_{k-1}+\tau\right)+\frac{1}{2} \Delta \dot{\theta}(\tau) \times \omega_{\mathrm{ab}}^{b}\left(t_{k-1}+\tau\right)+\frac{1}{2} \Delta \theta(\tau) \times \dot{\omega}_{\mathrm{ab}}^{b}\left(t_{k-1}+\tau\right) \\
\stackrel{\mathrm{t}}{\Phi}_{k}(\tau)=\frac{1}{2} \Delta \ddot{\theta}(\tau) \times \omega_{\mathrm{ab}}^{b}\left(t_{k-1}+\tau\right)+\Delta \dot{\theta}(\tau) \times \dot{\omega}_{\mathrm{ab}}^{b}\left(t_{k-1}+\tau\right) \\
\Phi_{k}^{(4)}(\tau)=\frac{3 \ddot{\theta}}{2}(\tau) \times \dot{\omega}_{\mathrm{ab}}^{b}\left(t_{k-1}+\tau\right) \\
\Phi_{k}^{(i)}(\tau)=0, \quad i=5,6,7, \ldots
\end{array}\right.
$$

According to (18) and (19), $\tau=0$ is substituted into (22); then, we have

$$
\left\{\begin{array}{l}
\dot{\Phi}_{k}(0)=\mathbf{a}, \\
\ddot{\Phi}_{k}(0)=2 \mathbf{b}, \\
\dot{t} \\
\Phi_{k}(0)=\mathbf{a} \times \mathbf{b}, \\
\Phi_{k}^{(i)}(0)=0, \quad i=4,5,6, \ldots
\end{array}\right.
$$

Substituting (23) into (19), we can get

$$
\Phi_{k}(T)=\Phi_{k}(0)+\mathbf{a} T+\mathbf{b} T^{2}+\frac{1}{6} \mathbf{a} \times \mathbf{b} T^{3},
$$

where $\Phi_{k}(0)$ is the rotation vector in the time period $T$. Since the time interval is $0, \Phi_{k}(0)=0$; therefore,

$$
\Phi_{k}(T)=\mathbf{a} T+\mathbf{b} T^{2}+\frac{1}{6} \mathbf{a} \times \mathbf{b} T^{3}
$$

From (14), the model coefficients $\mathbf{a}$ and $\mathbf{b}$ of the linear fitting of the vehicle angular velocity can be obtained by inverse solution; that is, 


$$
\left\{\begin{array}{l}
\mathbf{a}=\frac{3 \Delta \boldsymbol{\theta}_{1}-\Delta \boldsymbol{\theta}_{2}}{T}, \\
\mathbf{b}=\frac{2\left(\Delta \boldsymbol{\theta}_{2}-\Delta \boldsymbol{\theta}_{1}\right)}{T^{2}} .
\end{array}\right.
$$

Substituting (26) into (24), we can get

$$
\boldsymbol{\Phi}_{k}(T)=\Delta \boldsymbol{\theta}_{1}+\Delta \boldsymbol{\theta}_{2}+\frac{2}{3} \Delta \boldsymbol{\theta}_{1} \times \Delta \boldsymbol{\theta}_{2} .
$$

To solve the rotation vector according to (27), the angle increments $\Delta \theta_{1}$ and $\Delta \theta_{2}$ in the two time periods are used. Therefore, this formula is called the two-sample updating algorithm of the rotation vector.

Therefore, the solution of (16) by using the two-sample algorithm is as follows:

$$
\boldsymbol{\Phi}_{k}=\Delta \boldsymbol{\theta}_{1}+\Delta \boldsymbol{\theta}_{2}+\frac{2}{3} \Delta \boldsymbol{\theta}_{1} \times \Delta \boldsymbol{\theta}_{2} .
$$

Rotation vector $\Phi_{k}$ and its corresponding quaternion $\mathbf{q}_{b(k)}^{b(k-1)}$ are related as follows:

$$
\mathbf{q}_{b(k+1)}^{b(k)}=\cos \frac{\boldsymbol{\Phi}_{k}}{2}+\frac{\boldsymbol{\Phi}_{k}}{\boldsymbol{\Phi}} \sin \frac{\boldsymbol{\Phi}_{k}}{2} .
$$

Then, the calculated $\mathbf{q}_{b(k)}^{b(k-1)}$ is substituted into (16) to complete the attitude updating.

3.2. Velocity Updating Algorithm. When a carrier operates in the environment of line vibration and angular vibration, the sculling error may occur, which should not be ignored in a high-precision SINS algorithm.

The specific force given by (6) indicates the time parameters of each component as follows:

$$
\dot{V}^{a}(t)=\mathbf{R}_{b}^{a}(t) \mathbf{f}^{b}(t)+\mathbf{g}^{a}(t) .
$$

Equation (30) integrates in $\left[t_{k-1}, t_{k}\right]$ :

$$
\int_{t_{k-1}}^{t_{k}} \dot{V}^{a}(t) \mathrm{d} t=\int_{t_{k-1}}^{t_{k}}\left[\mathbf{R}_{b}^{a}(t) \mathbf{f}^{b}(t)+\mathbf{g}^{a}(t)\right] \mathrm{d} t .
$$

From (31),

$$
\mathbf{V}_{k}^{a}-\mathbf{V}_{k-1}^{a}=\int_{t_{k-1}}^{t_{k}} \mathbf{R}_{b}^{a}(t) \mathbf{f}^{b}(t) \mathrm{d} t+\int_{t_{k-1}}^{t_{k}} \mathbf{g}^{a}(t) \mathrm{d} t
$$

where $\mathbf{V}_{k}^{a}$ and $\mathbf{V}_{k-1}^{a}$ are velocities of the LCI frame at $t_{k}$ and $t_{k-1}$, respectively, and are recorded as

$$
\begin{aligned}
& \Delta \mathbf{V}_{\mathrm{sf}(k)}^{a}=\int_{t_{k-1}}^{t_{k}} \mathbf{R}_{b}^{a}(t) \mathbf{f}^{b}(t) \mathrm{d} t, \\
& \Delta \mathbf{V}_{g(k)}^{a}=\int_{t_{k-1}}^{t_{k}} \mathbf{g}^{a}(t) \mathrm{d} t,
\end{aligned}
$$

where $\Delta \mathbf{V}_{\mathrm{sf}(k)}^{a}$ and $\Delta \mathbf{V}_{g(k)}^{a}$ are the velocity increments of specific force and gravity in time period $T$, respectively. The following recurrence forms can be obtained:

$$
\mathbf{V}_{k}^{a}=\mathbf{V}_{k-1}^{a}+\Delta \mathbf{V}_{\mathrm{sf}(k)}^{a}+\Delta \mathbf{V}_{g(k)}^{a}
$$

The numerical integration algorithms for $\Delta \mathbf{V}_{\mathrm{sf}(k)}^{a}$ and $\Delta \mathbf{V}_{g(k)}^{a}$ are discussed below:

(1) First, we have the gravity velocity increment $\Delta \mathbf{V}_{g(k)}^{a}$.

Even for hypersonic vehicles, the change in the gravitational vector in a short time period $T$ is very small, so the integrand function of $\Delta \mathbf{V}_{g(k)}^{a}$ is generally considered to be a slow time variable, which can be replaced with the value at $t_{k-1 / 2}=\left(t_{k-1}+t_{k}\right) / 2$, and $\Delta \mathbf{V}_{g(k)}^{a}$ is approximated as follows:

$$
\Delta \mathbf{V}_{g(k)}^{a} \approx \mathbf{g}_{k-1 / 2}^{a} T .
$$

Since the navigation velocity and position at time $t_{k}$ are unknown, $\mathbf{g}_{k-1 / 2}^{a}$ needs to be calculated by linear extrapolation:

$$
\mathbf{x}_{k-1 / 2}=\mathbf{x}_{k-1}+\frac{\mathbf{x}_{k-1}-\mathbf{x}_{k-2}}{2}, \quad \mathbf{x}=\mathbf{g}^{a} .
$$

(2) Second, we have the specific force velocity increment $\Delta \mathbf{V}_{\mathrm{sf}(k)}^{a}$.

The integrand matrix of (33) is transformed to the matrix chain multiplication decomposition as follows.

$$
\begin{aligned}
\Delta \mathbf{V}_{\mathrm{sf}(k)}^{a} & =\int_{t_{k-1}}^{t_{k}} \mathbf{R}_{b(k-1)}^{a} \mathbf{R}_{b(t)}^{b(k-1)} \mathbf{f}^{b}(t) \mathrm{d} t \\
& =\mathbf{R}_{b(k-1)}^{a} \int_{t_{k-1}}^{t_{k}} \mathbf{R}_{b(t)}^{b(k-1)} \mathbf{f}^{b}(t) \mathrm{d} t .
\end{aligned}
$$

Hence,

$$
\Delta \mathbf{V}_{\mathrm{sf}(k)}^{b(k-1)}=\int_{t_{k-1}}^{t_{k}} \mathbf{R}_{b(t)}^{b(k-1)} \mathbf{f}^{b}(t) \mathrm{d} t
$$

For $t_{k-1} \leq t \leq t_{k}$, the relationship between the coordinate transformation matrix and the equivalent rotation vector is as follows:

$$
\mathbf{R}_{b(t)}^{b(k-t)}=I+\frac{\sin \Phi}{\Phi}(\boldsymbol{\Phi} \times)+\frac{1-\cos \Phi}{\Phi^{2}}(\boldsymbol{\Phi} \times)(\boldsymbol{\Phi} \times),
$$

Equation (42) can be approximated as

$$
\begin{array}{r}
\frac{\sin \Phi}{\Phi} \approx 1, \\
\frac{1-\cos \Phi}{\Phi^{2}} \approx \frac{1}{2},
\end{array}
$$

$(\Phi \times)(\Phi \times)$ can be regarded as a second-order small quantity. Equations (20) and (40) can be approximated as

$$
\mathbf{R}_{b(t)}^{b(k-1)}=\mathbf{I}+(\Delta \boldsymbol{\theta} \times) .
$$

By substituting (42) into (39), we obtain 


$$
\Delta \mathbf{V}_{s f(k)}^{b}=\int_{t_{k-1}}^{t_{k}}\left[\mathbf{f}^{b}(t)+\Delta \boldsymbol{\theta} \times \mathbf{f}^{b}(t)\right] \mathrm{d} t
$$

Hence,

$$
\Delta \mathbf{V}_{k}=\int_{t_{k-1}}^{t_{k}} \mathbf{f}^{b}(t) \mathrm{d} t
$$

$$
\begin{aligned}
& \text { Since } \\
& \frac{\mathrm{d}}{\mathrm{d} t}[\Delta \boldsymbol{\theta}(t) \times \Delta \mathbf{V}(t)]=\Delta \boldsymbol{\theta} \times \Delta \dot{V}(t)-\Delta \mathbf{V}(t) \times \Delta \dot{\theta}(t),
\end{aligned}
$$

the following formula can be obtained:

From (14),

$$
\left\{\begin{array}{l}
\Delta \dot{\theta}(t)=\boldsymbol{\omega}_{\mathrm{ab}}^{b}(t), \\
\Delta \dot{V}(t)=\mathbf{f}^{b}(t) .
\end{array}\right.
$$

$$
\begin{aligned}
\Delta \boldsymbol{\theta}(t) \times \mathbf{f}^{b}(t) & =\Delta \boldsymbol{\theta}(t) \times \Delta \dot{V}(t)=\frac{\mathrm{d}}{\mathrm{d} t}[\Delta \boldsymbol{\theta}(t) \times \Delta \mathbf{V}(t)]+\Delta \mathbf{V}(t) \times \Delta \dot{\theta}(t) \\
& =\frac{1}{2} \frac{\mathrm{d}}{\mathrm{d} t}[\Delta \boldsymbol{\theta}(t) \times \Delta \mathbf{V}(t)]+\frac{1}{2}\left[\Delta \boldsymbol{\theta}(t) \mathbf{f}^{b}(t)+\Delta \mathbf{V}(t) \times \boldsymbol{\omega}_{\mathrm{ab}}^{b}(t)\right]
\end{aligned}
$$

Equations (44) and (47) are substituted into (43), when

Hence,

$t=t_{k+1}, \Delta \mathbf{V}\left(t_{k-1}\right)=0$, and $\Delta \theta\left(t_{k-1}\right)=0$ and when

$t=t_{k}, \Delta \mathbf{V}\left(t_{k}\right)=\Delta \mathbf{V}_{k}$, and $\Delta \theta\left(t_{k}\right)=\Delta \theta_{k}$.

$$
\begin{aligned}
\Delta \mathbf{V}_{s f(k)}^{b(k-1)} & =\Delta \mathbf{V}_{k}+\frac{1}{2} \Delta \boldsymbol{\theta}_{k} \times \Delta \mathbf{V}_{k}+\frac{1}{2} \int_{t_{k-1}}^{t_{k}}\left[\Delta \boldsymbol{\theta}(t) \times \mathbf{f}^{b}(t)+\Delta \mathbf{V}(t) \times \boldsymbol{\omega}_{\mathrm{ab}}^{b}(t)\right] \mathrm{d} t \\
& =\Delta \mathbf{V}_{k}+\Delta \mathbf{V}_{\text {rotk }}+\Delta \mathbf{V}_{\text {sculk }},
\end{aligned}
$$

where

$$
\Delta \mathbf{V}_{\text {rotk }}=\frac{1}{2} \Delta \boldsymbol{\theta}_{k} \times \Delta \mathbf{V}_{k}
$$

Here, $\Delta \mathbf{V}_{\text {rotk }}$ is the compensation quantity of the ve$\Delta \mathbf{V}_{\text {sculk }}$ is the compensation term of the velocity sculling effect when the carrier has linear and angular vibrations.

Equations (12) and (13) are substituted into (50) and locity rotation effect, caused by the rotation of the linear motion direction of the carrier.

$\Delta \mathbf{V}_{\text {sculk }}=\frac{1}{2} \int_{t_{k-1}}^{t_{k}}\left[\Delta \boldsymbol{\theta}(t) \times \mathbf{f}^{b}(t)+\Delta \mathbf{V}(t) \times \boldsymbol{\omega}_{\mathrm{ab}}^{b}(t)\right] \mathrm{d} t$,

$$
\begin{aligned}
\Delta \mathbf{V}_{\text {sculk }}= & \frac{1}{2} \int_{t_{k-1}}^{t_{k}}\left[\left(\mathbf{a}\left(t-t_{k-1}\right)+\mathbf{b}\left(t-t_{k-1}\right)^{2}\right) \times\left(\mathbf{A}+2 \mathbf{B}\left(t-t_{k-1}\right)\right)\right. \\
& \left.+\left(\mathbf{A}\left(t-t_{k-1}\right)+\mathbf{B}\left(t-t_{k-1}\right)^{2}\right) \times\left(\mathbf{a}+2 \mathbf{b}\left(t-t_{k-1}\right)\right)\right] d t \\
= & (\mathbf{a} \times \mathbf{B}+\mathbf{A} \times \mathbf{b}) \frac{\left(t_{k}-t_{k-1}\right)^{3}}{6} .
\end{aligned}
$$


From (14),

$$
\left\{\begin{array}{l}
\mathbf{A}=\frac{3 \Delta \mathbf{V}_{1}-\Delta \mathbf{V}_{2}}{2 h}, \\
\mathbf{B}=\frac{\Delta \mathbf{V}_{2}-\Delta \mathbf{V}_{1}}{2 h^{2}} .
\end{array}\right.
$$

By substituting (26) and (52) into (51), the two-sample algorithm for the compensation of the velocity sculling error is obtained as follows:

$$
\begin{aligned}
\Delta \mathbf{V}_{\text {sculk }} & =\left(\frac{3 \Delta \boldsymbol{\theta}_{1}-\Delta \boldsymbol{\theta}_{2}}{2 h} \times \frac{\Delta \mathbf{V}_{2}-\Delta \mathbf{V}_{1}}{2 h^{2}}+\frac{3 \Delta \mathbf{V}_{1}-\Delta \mathbf{V}_{2}}{2 h} \times \frac{\Delta \boldsymbol{\theta}_{2}-\Delta \boldsymbol{\theta}_{1}}{2 h^{2}}\right) \frac{(2 h)^{3}}{6} \\
& =\frac{2}{3}\left(\Delta \boldsymbol{\theta}_{1} \times \Delta \mathbf{V}_{2}+\Delta \mathbf{V}_{1} \times \Delta \boldsymbol{\theta}_{2}\right) .
\end{aligned}
$$

Thus, velocity updating algorithm derivation is completed.

3.3. Position Updating Algorithm. When the hypersonic vehicle is in sculling flight, a scrolling effect error is present in the solution of the SINS position. According to (35) and (36),

$$
\mathbf{V}^{a}(t)=\mathbf{V}_{k-1}^{a}+\Delta \mathbf{V}_{\mathrm{sf}}^{a}(t)+\Delta \mathbf{V}_{g}^{a} \frac{t-t_{k-1}}{T}, \quad t_{k-1} \leq t \leq t_{k},
$$

$$
\Delta \mathbf{V}_{s f(k)}^{a}=\int_{t_{k-1}}^{t_{k}} \mathbf{R}_{b}^{a}(t) \mathbf{f}^{b}(t) \mathrm{d} t .
$$

By integrating (54) in time period $\left[t_{k-1}, t_{k}\right]$, we get

$$
\Delta \mathbf{P}_{k}^{a}=\left[\mathbf{V}_{k-1}^{a}+\frac{1}{2} \Delta \mathbf{V}_{g(k)}^{a}\right] T+\Delta \mathbf{P}_{s f(k)}^{a} .
$$

From (38), we obtain

$$
\Delta \mathbf{P}_{\mathrm{sf}(k)}^{a}=\int_{t_{k-1}}^{t_{k}} \Delta \mathbf{V}_{\mathrm{sf}(k)}^{a} \mathrm{~d} t=\mathbf{R}_{b(k-1)}^{a} \int_{t_{k-1}}^{t_{k}} \Delta \mathbf{V}_{\mathrm{sf}(k)}^{b(k-1)} \mathrm{d} t .
$$

Using (48), the following can be calculated:

where

$$
\Delta \mathbf{P}_{\mathrm{sf}(k)}^{b}=\int_{t_{k-1}}^{t_{k}} \Delta \mathbf{V}_{\mathrm{sf}(k)}^{b(k-1)} \mathrm{d} t=\int_{t_{k-1}}^{t_{k}}\left[\Delta \mathbf{V}(t)+\frac{1}{2} \Delta \boldsymbol{\theta}^{b}(t) \times \Delta \mathbf{V}^{b}(t)+\Delta \mathbf{V}_{\mathrm{scul}}(t)\right] \mathrm{d} t
$$

Note that $\gamma_{1}=1 / 2 \int_{t_{k-1}}^{t_{k}} \Delta \theta^{b}(t) \times \Delta \mathbf{V}^{b}(t) \mathrm{d} t$.

The above integral is obtained by a step integral method:

$$
\begin{aligned}
& \gamma_{2}=\frac{1}{2} \int_{t_{k-1}}^{t_{k}} \Delta \boldsymbol{\theta}^{b}(t) \times \Delta \mathbf{V}^{b}(t) \mathrm{d} t=\frac{1}{2} \mathbf{S}_{\Delta \theta k}^{b} \times \Delta \mathbf{V}_{k}^{b}-\frac{1}{2} \int_{t_{k-1}}^{t_{k}} b_{\Delta \theta}^{b}(t) \times \mathbf{f}^{b}(t) \mathrm{d} t, \\
& \gamma_{3}=\frac{1}{2} \int_{t_{k-1}}^{t_{k}} \Delta \boldsymbol{\theta}^{b}(t) \times \Delta \mathbf{V}^{b}(t) \mathrm{d} t=\frac{1}{2} \boldsymbol{\theta}_{k}^{b} \times \mathbf{S}_{\Delta v_{k}}^{b}+\frac{1}{2} \int_{t_{k-1}}^{t_{k}} \mathbf{S}_{\Delta v}^{b}(t) \times \boldsymbol{\omega}_{\mathrm{ab}}^{b}(t) \mathrm{d} t .
\end{aligned}
$$

Thus,

$$
\begin{aligned}
\frac{1}{2} \int_{t_{k-1}}^{t_{k}} \Delta \boldsymbol{\theta}^{b}(t) \times \Delta \mathbf{V}^{b}(t) \mathrm{d} t & =\frac{1}{3}\left(\gamma_{1}+\gamma_{2}+\gamma_{3}\right) \\
& =\frac{1}{6}\left(\mathbf{S}_{\Delta \theta k}^{b} \times \Delta \mathbf{V}_{k}^{b}+\frac{1}{2} \boldsymbol{\theta}_{k}^{b} \times \mathbf{S}_{\Delta v k}^{b}\right)+\frac{1}{6} \int_{t_{k-1}}^{t_{k}}\left[\mathbf{S}_{\Delta v}^{b}(t) \times \boldsymbol{\omega}_{\mathrm{ab}}^{b}(t)-\mathbf{S}_{\Delta \theta}^{b}(t) \times \mathbf{f}^{b}(t)+\Delta \boldsymbol{\theta}^{b}(t) \times \Delta \mathbf{V}^{b}(t)\right] \mathrm{d} t
\end{aligned}
$$


The above equation is substituted into (58) to obtain

$$
\Delta \mathbf{P}_{\mathrm{sf}(k)}^{b}=\int_{t_{k-1}}^{t_{k}} \Delta \mathbf{V}_{\mathrm{sf}(k)}^{b} \mathrm{~d} t=\mathbf{S}_{\Delta v k}^{b}+\Delta \mathbf{P}_{\text {rotk }}^{b}+\Delta \mathbf{P}_{\text {scrlk }}^{b},
$$

where

$$
\mathbf{S}_{\Delta v k}^{b}=\int_{t_{k-1}}^{t_{k}} \Delta \mathbf{V}(t) \mathrm{d} t=\int_{t_{k-1}}^{t_{k}} \int_{t_{k-1}}^{t_{k}} \mathbf{f}^{b}(\boldsymbol{\tau}) \mathrm{d} \tau \mathrm{d} t
$$

That is, the second integral increment of specific force is obtained.

$$
\Delta \mathbf{P}_{\text {rotk }}^{b}=\frac{1}{6}\left(\mathbf{S}_{\Delta \theta k}^{b} \times \Delta \mathbf{V}_{k}^{b}+\Delta \boldsymbol{\theta}_{k}^{b} \times \mathbf{S}_{\Delta v k}^{b}\right) .
$$

Equation (63) is the compensation of the rotation effect in position calculation.

$$
\Delta \mathbf{P}_{\text {scrlk }}^{b}=\frac{1}{6} \int_{t_{k-1}}^{t_{k}}\left[\mathbf{S}_{\Delta v}^{b}(t) \times \boldsymbol{\omega}_{a b}^{b}(t)-\mathbf{S}_{\Delta \theta}^{b}(t) \times \mathbf{f}^{b}(t)+\Delta \boldsymbol{\theta}^{b}(t) \times \Delta \mathbf{V}^{b}(t)+6 \Delta \mathbf{V}_{\text {scul }}(t)\right] \mathrm{d} t .
$$

Equation (64) is the compensation of the scrolling effect in position calculation, and it indicates that the factors affecting the scrolling effect are the sculling effect and the coupling effect between the angular and linear motions of the vehicle.

From (12) and (13), we obtain the following:

$$
\begin{aligned}
& \Delta \boldsymbol{\theta}_{k}=\int_{t_{k-1}}^{t_{k}} \boldsymbol{\omega}_{\mathrm{ab}}^{b}(t) \mathrm{d} t=T \mathbf{a}+T^{2} \mathbf{b}, \\
& \Delta \mathbf{V}_{k}=\int_{t_{k-1}}^{t_{k}} \mathbf{f}(t) \mathrm{d} t=T \mathbf{A}+T^{2} \mathbf{B},
\end{aligned}
$$

$$
\begin{gathered}
\mathbf{S}_{\Delta \theta k}=\int_{t_{k-1}}^{t_{k}} \int_{t_{k-1}}^{\tau} \boldsymbol{\omega}_{\mathrm{ab}}^{b}(\mu) \mathrm{d} \mu \mathrm{d} \tau=\frac{T^{2}}{2} \mathbf{a}+\frac{T^{3}}{3} \mathbf{b}, \\
\mathbf{S}_{\Delta v k}=\int_{t_{k-1}}^{t_{k}} \int_{t_{k-1}}^{\tau} \mathbf{f}(\mu) \mathrm{d} \mu \mathrm{d} \tau=\frac{T^{2}}{2} \mathbf{A}+\frac{T^{3}}{3} \mathbf{B} .
\end{gathered}
$$

Equations (26) and (52) as well as (65)-(68) are substituted into (62)-(64) to obtain the following.

$$
\begin{aligned}
\mathbf{S}_{\Delta v k}^{b} & =T\left[\frac{5}{6} \Delta \mathbf{V}_{1}+\frac{1}{6} \Delta \mathbf{V}_{2}\right] \\
\Delta \mathbf{P}_{\text {rotk }}^{b} & =T\left(\Delta \boldsymbol{\theta}_{1} \times\left[\frac{5}{18} \Delta \mathbf{V}_{1}+\frac{1}{6} \Delta \mathbf{V}_{2}\right]+\Delta \boldsymbol{\theta}_{2} \times\left[\frac{1}{6} \Delta \mathbf{V}_{1}+\frac{1}{18} \Delta \mathbf{V}_{2}\right]\right) \\
\Delta \mathbf{P}_{\text {scrlk }}^{b}= & T\left(\Delta \boldsymbol{\theta}_{1} \times\left[\frac{11}{90} \Delta \mathbf{V}_{1}+\frac{1}{10} \Delta \mathbf{V}_{2}\right]+\Delta \boldsymbol{\theta}_{2} \times\left[\frac{1}{90} \Delta V_{2}-\frac{7}{30} \Delta \mathbf{V}_{1}\right]\right) \\
\dot{\widehat{R}}_{b}^{a} & =\widehat{R}_{b}^{a} \widehat{\Omega}_{\mathrm{ab}}^{b} .
\end{aligned}
$$

Thus, position updating algorithm derivation is completed.

\section{SINS/BDS Integrated Navigation Algorithm in the LCI Frame}

\subsection{SINS Error Equation in the Launch-Centered Inertial Frame}

4.1.1. Attitude Error Equation. For the SINS, the transformation matrix error from the BF frame to the calculated frame is caused by the angular rate error between the two coordinate systems when they are rotated. The attitude mechanization in the LCI frame is as follows.

$$
\dot{R}_{b}^{a}=\mathbf{R}_{b}^{a} \mathbf{\Omega}_{\mathrm{ab}}^{b} .
$$

Considering the measurement and calculation errors, the transformation rate of the calculated transformation matrix is given as follows.
The calculated transformation matrix $\widehat{\mathbf{R}}_{b}^{a}$ can be written as

$$
\widehat{R}_{b}^{a}=\mathbf{R}_{b}^{a}+\delta \mathbf{R}_{b}^{a} .
$$

If

$$
\delta \mathbf{R}_{b}^{a}=-\Psi^{a} \mathbf{R}_{b}^{a}
$$

then

$$
\widehat{R}_{b}^{a}=\left(\mathbf{I}-\Psi^{a}\right) \mathbf{R}_{b}^{a} .
$$

There is an error angle $\varphi^{a}=\left[\begin{array}{lll}\phi_{x} & \phi_{y} & \phi_{z}\end{array}\right]^{T}$ between the actual navigation frame and the calculated frame, and $\Psi^{a}$ is the antisymmetric matrix of $\varphi^{a}$ :

$$
\boldsymbol{\Psi}^{a}=\left[\begin{array}{ccc}
0 & \phi_{z} & -\phi_{y} \\
-\phi_{z} & 0 & \phi_{x} \\
\phi_{y} & -\phi_{x} & 0
\end{array}\right] .
$$


Derivation of both sides of (74) gives the following:

$$
\begin{aligned}
\dot{\hat{R}}_{b}^{a} & =\dot{R}_{b}^{a}-\dot{\Psi}^{a} \mathbf{R}_{b}^{a}-\Psi^{a} \dot{R}_{b}^{a}, \\
\delta \dot{R}_{b}^{a} & =-\dot{\Psi}^{a} \mathbf{R}_{b}^{a}-\Psi^{a} \dot{R}_{b}^{a} .
\end{aligned}
$$
as

By contrast, the differential equation of (70) is obtained

$\delta \dot{R}_{b}^{a}=\delta \mathbf{R}_{b}^{a} \boldsymbol{\Omega}_{\mathrm{ab}}^{b}+\mathbf{R}_{b}^{a} \delta \boldsymbol{\Omega}_{\mathrm{ab}}^{b}=-\Psi^{a} \mathbf{R}_{b}^{a} \boldsymbol{\Omega}_{\mathrm{ab}}^{b}+\mathbf{R}_{b}^{a} \delta \boldsymbol{\Omega}_{\mathrm{ab}}^{b}$.

Comparing (76) and (77), we get

$$
\dot{\Psi}^{a}=-\mathbf{R}_{b}^{a} \delta \mathbf{\Omega}_{\mathrm{ab}}^{b} \mathbf{R}_{a}^{b} .
$$

Equation (78) is written in a vector form as

$$
\dot{\varphi}^{a}=-\mathbf{R}_{b}^{a} \delta \omega_{\mathrm{ab}}^{b} .
$$

The gyroscope measurement error is $\delta \omega_{\mathrm{ab}}^{b}=\delta \omega_{\mathrm{ai}}^{b}+\delta \omega_{\mathrm{ib}}^{b}$, and because $\delta \omega_{\mathrm{ai}}^{b}=0, \delta \omega_{\mathrm{ib}}^{b}$ is assumed to be composed of the random constant drift $\varepsilon^{b}$ of the equivalent gyroscope. Thus,

$$
\dot{\varphi}^{a}=-\mathbf{R}_{b}^{a} \boldsymbol{\varepsilon}^{b} .
$$

Therefore, the attitude error equation is written in the component form as

$$
\left[\begin{array}{c}
\dot{\phi}_{x}^{a} \\
\dot{\phi}_{y}^{a} \\
\dot{\phi}_{z}^{a}
\end{array}\right]=-\mathbf{R}_{a}^{b}\left[\begin{array}{c}
\varepsilon_{x}^{b} \\
\varepsilon_{y}^{b} \\
\varepsilon_{z}^{b}
\end{array}\right] .
$$

4.1.2. Velocity Error Equation. From (6), the linear equation of the first-order small disturbance for velocity can be obtained as

$$
\delta \dot{V}^{a}=\delta \mathbf{R}_{b}^{a} \mathbf{f}^{b}+\mathbf{R}_{b}^{a} \delta \mathbf{f}^{b}+\delta \mathbf{g}^{a} .
$$

The measurement error $\delta \mathbf{f}^{b}$ of the accelerometer consists of the random constant zero bias $\nabla^{b}$ of the equivalent accelerometer.

Therefore, we can get the velocity error equation as follows.

$$
\delta \dot{V}^{a}=\delta \mathbf{R}_{b}^{a} \mathbf{f}^{b}+\delta \mathbf{g}^{a}+\mathbf{R}_{b}^{a} \nabla^{b}
$$

By substituting (73) into (83), we get

$$
\delta \dot{V}^{a}=-\Psi \mathbf{R}_{b}^{a} \mathbf{f}^{b}+\delta \mathbf{g}^{a}+\mathbf{R}_{b}^{a} \nabla^{b}=\mathbf{F}^{a} \boldsymbol{\varphi}^{a}+\delta \mathbf{g}^{a}+\mathbf{R}_{b}^{a} \nabla^{b},
$$

where

$$
\delta \mathbf{g}^{a}=\frac{u}{r^{3}}\left[\begin{array}{ccc}
3 \frac{x^{2}}{r^{2}}-1 & 3 \frac{x y}{r^{2}} & 3 \frac{x z}{r^{2}} \\
3 \frac{y x}{r^{2}} & 3 \frac{y^{2}}{r^{2}}-1 & 3 \frac{y z}{r^{2}} \\
3 \frac{z x}{r^{2}} & 3 \frac{z y}{r^{2}} & 3 \frac{z^{2}}{r^{2}}-1
\end{array}\right]\left[\begin{array}{l}
\delta x \\
\delta y \\
\delta z
\end{array}\right]=\mathbf{G}_{P}^{a} \delta \mathbf{P} .
$$

4.1.3. Position Error Equation. The position error equation under the LCI frame is

$$
\delta \dot{P}^{a}=\delta \mathbf{V}^{a} .
$$

4.2. SINS/BDS Integrated Navigation Equation. The state and measurement equation for SINS/BDS integrated navigation are given as follows.

$$
\begin{aligned}
\dot{X} & =\mathbf{F X}+\mathbf{G W}, \\
\mathbf{Z}_{\mathrm{vp}} & =\mathbf{H}_{\mathrm{vp}} \mathbf{X}+\mathbf{V}_{\mathrm{vp}},
\end{aligned}
$$

where $\mathbf{Z}_{\mathrm{vp}}$ is shown in

$$
\mathbf{Z}_{\mathrm{vp}}(t)=\left[\begin{array}{l}
\mathbf{V}^{a}-\mathbf{V}_{s}^{a} \\
\mathbf{P}^{a}-\mathbf{P}_{s}^{a}
\end{array}\right] .
$$

It is assumed that the measurement error $\delta \omega_{\mathrm{ab}}^{b}$ of the gyroscope is composed of the gyroscope's zero bias $\varepsilon^{b}$ and that the measurement error $\delta \mathbf{f}^{b}$ of the accelerometer consists of the accelerometer's zero bias $\nabla^{b}$. The state variable of SINS/BDS is adopted; that is,

$$
\mathbf{X}=\left[\begin{array}{lllll}
\boldsymbol{\varphi}^{a} & \delta \mathbf{V}^{a} & \delta \mathbf{P}^{a} & \boldsymbol{\varepsilon}^{b} & \nabla^{b}
\end{array}\right]^{T} .
$$

The state equation is

$$
\left[\begin{array}{c}
\phi^{a} \\
\delta \dot{V}^{a} \\
\delta \dot{P}^{a} \\
\dot{\varepsilon}^{b} \\
\dot{\nabla}^{b}
\end{array}\right]=\left[\begin{array}{ccccc}
0_{3 \times 3} & 0_{3 \times 3} & 0_{3 \times 3} & -\mathbf{R}_{b}^{a} & 0_{3 \times 3} \\
\mathbf{F}^{a} & 0_{3 \times 3} & G_{P}^{a} & 0_{3 \times 3} & \mathbf{R}_{b}^{a} \\
0_{3 \times 3} & \mathbf{I}_{3 \times 3} & 0_{3 \times 3} & 0_{3 \times 3} & 0_{3 \times 3} \\
0_{3 \times 3} & 0_{3 \times 3} & 0_{3 \times 3} & 0_{3 \times 3} & 0_{3 \times 3} \\
0_{3 \times 3} & 0_{3 \times 3} & 0_{3 \times 3} & 0_{3 \times 3} & 0_{3 \times 3}
\end{array}\right]\left[\begin{array}{c}
\phi^{a} \\
\delta \mathbf{V}^{a} \\
\delta \mathbf{P}^{a} \\
\boldsymbol{\varepsilon}^{b} \\
\nabla^{b}
\end{array}\right]+\left[\begin{array}{cc}
-\mathbf{R}_{b}^{a} & 0_{3 \times 3} \\
0_{3 \times 3} & \mathbf{R}_{b}^{a} \\
0_{9 \times 3} & 0_{9 \times 3}
\end{array}\right]\left[\begin{array}{c}
\mathbf{w}_{g} \\
\mathbf{w}_{g}
\end{array}\right] .
$$


The 15-dimensional measurement matrix $\mathbf{H}_{\mathrm{vp}}$ is expressed as follows:

$$
\mathbf{H}_{\mathrm{vp}}=\left[\begin{array}{ccccc}
0_{3 \times 3} & \mathbf{I}_{3 \times 3} & 0_{3 \times 3} & 0_{3 \times 3} & 0_{3 \times 3} \\
0_{3 \times 3} & 0_{3 \times 3} & \mathbf{I}_{3 \times 3} & 0_{3 \times 3} & 0_{3 \times 3}
\end{array}\right] .
$$

4.3. BDS Receiver Position and Velocity Conversion. Since the BDS receiver outputs the position and velocity in the ECEF frame [20], they need to be converted to the LCI frame. Let the position and velocity of the BDS receiver in the ECEF frame be $\mathbf{P}_{s}^{e}$ and $\mathbf{V}_{s}^{e}$, respectively. First, by converting these values in to the LECI frame, position $\mathbf{P}_{s}^{t}$ and velocity $\mathbf{V}_{s}^{t}$ in the LECI are obtained as shown in

$$
\begin{aligned}
& \mathbf{P}_{s}^{t}=\mathbf{R}_{z}\left(\omega_{\mathrm{ie}} t\right) \mathbf{P}_{s}^{e}, \\
& \mathbf{V}_{s}^{t}=\mathbf{R}_{z}\left(\omega_{\mathrm{ie}} t\right)\left(\mathbf{V}_{s}^{e}+\left[\begin{array}{c}
0 \\
0 \\
\omega_{\mathrm{ie}}
\end{array}\right] \times \mathbf{P}_{s}^{e}\right)
\end{aligned}
$$

Then, position $\mathbf{P}_{s}^{a}$ and velocity $\mathbf{V}_{s}^{a}$ of the BDS receiver in the LCI frame are obtained by converting position $\mathbf{P}_{s}^{t}$ and velocity $\mathbf{V}_{s}^{t}$ from the LECI frame to the LCI frame, as shown in

$$
\begin{aligned}
& \mathbf{P}_{s}^{a}=\mathbf{R}_{t}^{a}\left(\mathbf{P}_{s}^{t}-\mathbf{P}_{0}^{t}\right), \\
& \mathbf{V}_{s}^{a}=\mathbf{R}_{t}^{a} \mathbf{V}_{s}^{t} .
\end{aligned}
$$

\section{Test on SINS/BDS Integrated Navigation}

5.1. SINS/BDS Integrated Navigation Vehicle Field Test. To test the feasibility of the SINS/BDS integrated navigation algorithm, a vehicle field test was conducted, as shown in Figure 2. The test was conducted at a high speed around Xi'an, and the track of the vehicle is shown in Figure 3. The initial latitude and longitude of the vehicle test were $34.19785^{\circ}$ and $108.8284^{\circ}$, respectively, the initial height was $365.1 \mathrm{~m}$, and the initial velocity was $0 \mathrm{~m} / \mathrm{s}$. The vehicle was equipped with a set of high-precision integrated navigation system as the reference, called the master INS. Its main performance indicators are as follows: position accuracy is $1.0 \mathrm{nmile} / \mathrm{h}$, speed accuracy is $0.8 \mathrm{~m} / \mathrm{s}$, and attitude accuracy is $0.05^{\circ}$.

The SINS/BDS used in this paper is called the slave INS. The gyroscope drift was $3^{\circ} / \mathrm{h}(1 \sigma)$, the scale factor was $100 \mathrm{ppm}$, the variance of white noise was $0.3^{\circ} / \mathrm{h}(1 \sigma)$, and the output period of gyroscope was $2 \mathrm{~ms}$. The bias of the accelerometer was $1 \times 10^{-3} \cdot \mathrm{g}(1 \sigma)$, the scale factor was $100 \mathrm{ppm}$, the white noise variance was $1 \times 10^{-4} \cdot \mathrm{g}(1 \sigma)$, and the output period was $2 \mathrm{~ms}$. The positioning accuracy of the BDS satellite receiver was $10 \mathrm{~m}(1 \sigma)$, the speed accuracy was $0.3 \mathrm{~m} / \mathrm{s}(1 \sigma)$, and the output period was $1 \mathrm{~Hz}$.

The master INS output the navigation solutions were in the local horizontal frame. The slave INS solutions were converted from the LCI frame to the local horizontal frame

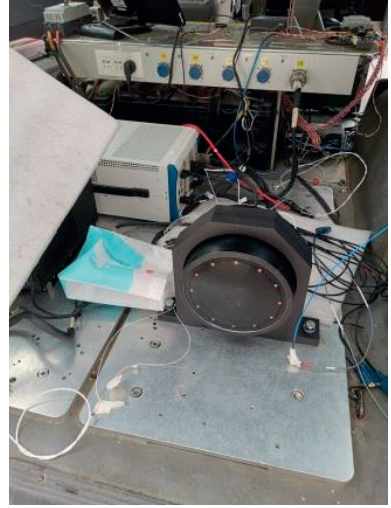

(a)

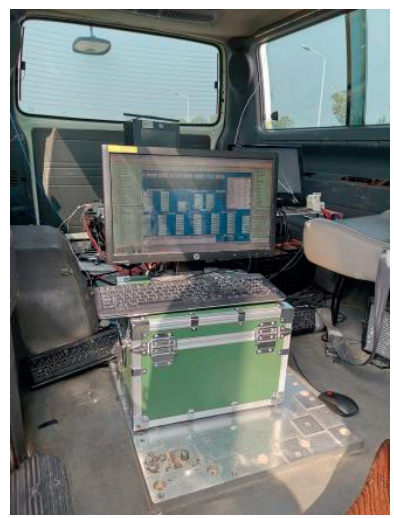

(b)
FIGURE 2: SINS/BDS integrated navigation system.

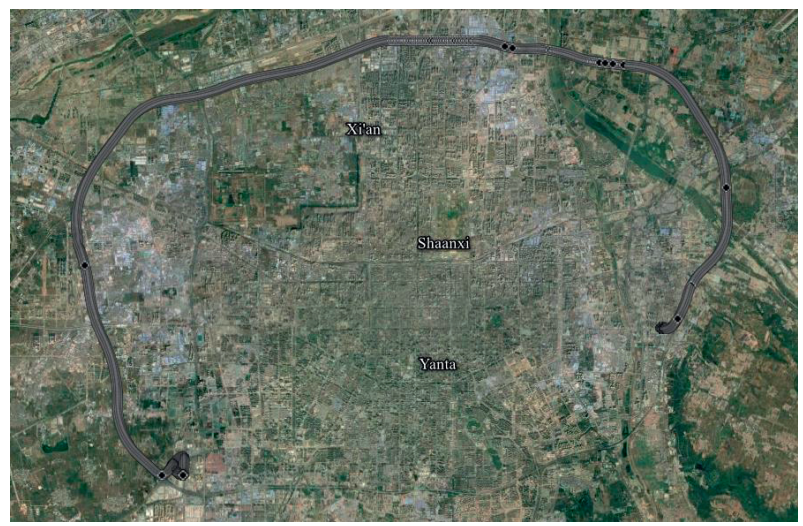

Figure 3: Test track of vehicle.

[21]. Both are then compared and made the difference as shown in Figures 4-10.

In the vehicle test, the azimuth angle error of SINS/BDS integrated navigation was approximately $0.05^{\circ}$, the pitch angle error was within $0.05^{\circ}$, the roll angle error was within $0.05^{\circ}$, the velocity error of east and north was within $0.2 \mathrm{~m} / \mathrm{s}$, and the position error was within $10 \mathrm{~m}$.

5.2. SINS/BDS Integrated Navigation Test by HWIL Simulation. Due to the limitation of vehicle velocity, the performance of SINS/BDS integrated navigation was verified for hypersonic velocity by the HWIL simulation of hypersonic vehicles [4], as shown in Figure 11. The HWIL simulation equipment includes the real-time simulator, GNC analysis computer, 3D display, three-axis rotation table, IMU simulator, GNSS simulator, product interface system, and actuator load simulator. The on-board equipment includes the on-board computer, SINS/BDS system, and actuator system.

We propose a classic boost-glide flight trajectory $[22,23]$. The equation describing this trajectory is detailed in [22], and the trajectory data are obtained from the simulation platform in [23]. The initial parameters of the 


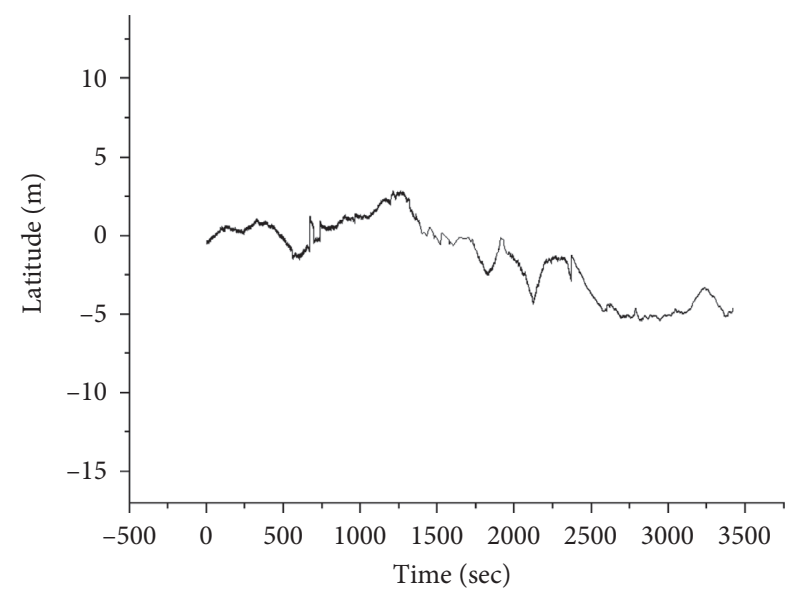

FIgURE 4: Error of master and slave SINS/BDS latitude.

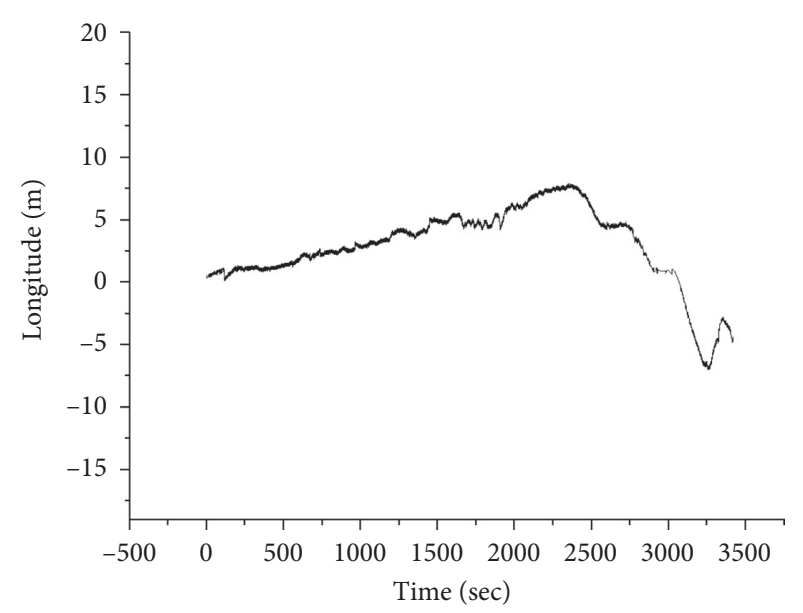

FIGURE 5: Error of master and slave SINS/BDS longitude.

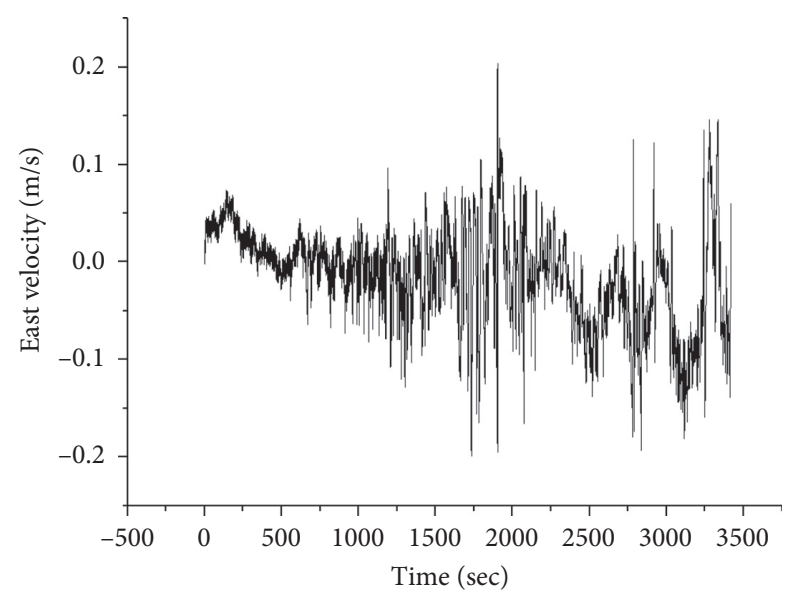

FIGURE 6: Error of master and slave SINS/BDS east velocity.

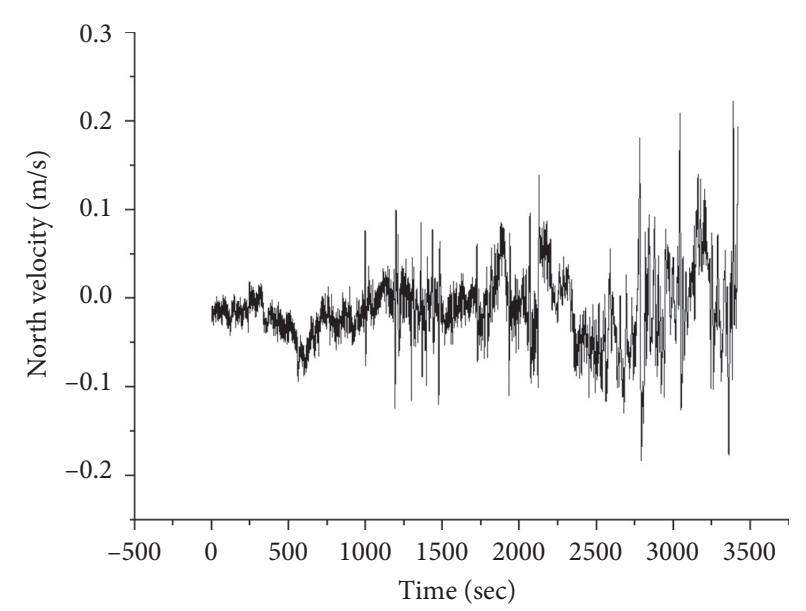

FIGURE 7: Error of master and slave SINS/BDS north velocity.

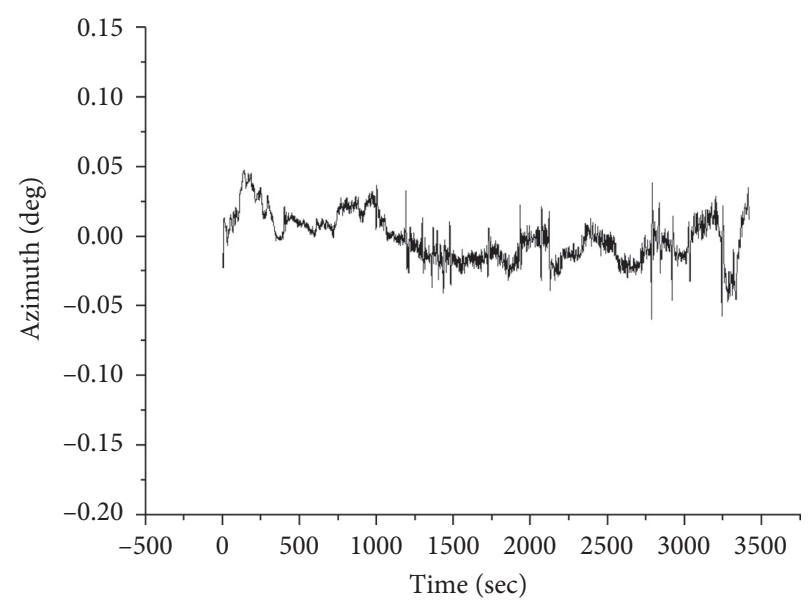

FIGURE 8: Error of master and slave SINS/BDS azimuth angle.

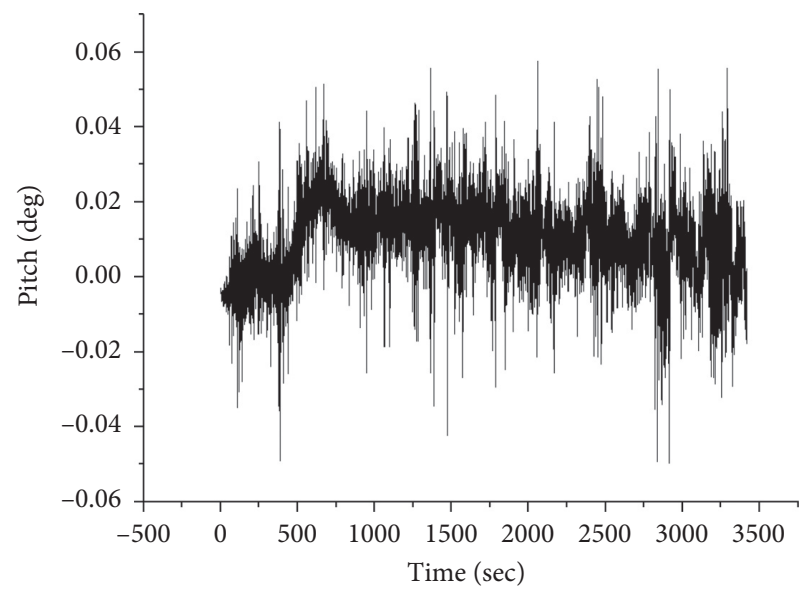

FIGURE 9: Error of master and slave SINS/BDS pitch angle. 


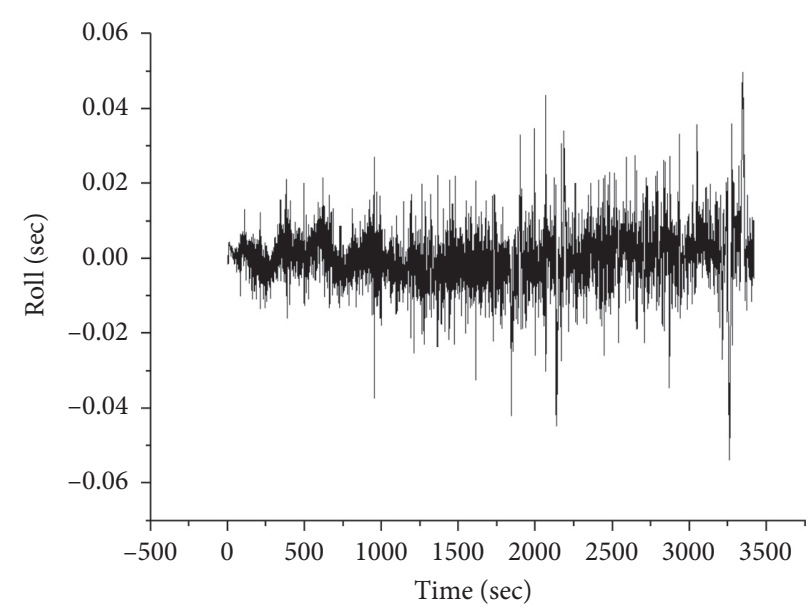

FIGURE 10: Error of master and slave SINS/BDS roll angle.

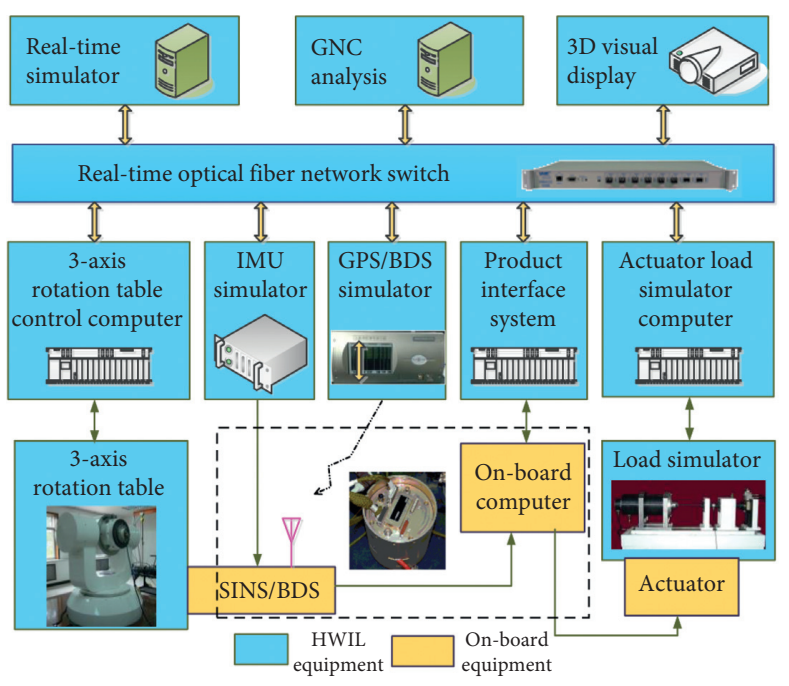

Figure 11: Diagram of HWIL simulation system.

TABLE 1: Initial state of the trajectory.

\begin{tabular}{lc}
\hline Parameters & Value \\
\hline Velocity & $0 \mathrm{~m} / \mathrm{s}$ \\
Latitude & $34.2^{\circ}$ \\
Longitude & $108.9^{\circ}$ \\
Height & $0 \mathrm{~m}$ \\
Launch azimuth & $200^{\circ}$ \\
Pitch & $90^{\circ}$ \\
Roll & $0^{\circ}$ \\
Yaw & $0^{\circ}$ \\
\hline
\end{tabular}

trajectory are shown in Table 1, and the simulation diagram of the trajectory is shown in Figure 12.

The simulation results presented in Figures 13-17 indicate that the three attitude angle errors of the SINS/BDS integrated navigation system can basically converge within 0.05 . The velocity error in the three directions is within $0.2 \mathrm{~m} / \mathrm{s}$. The position error in the three directions is less than $10 \mathrm{~m}$. After $200 \mathrm{~s}$, the gyroscope drift in all three directions is estimated to be $3.1^{\circ} / \mathrm{h}$. The drift of accelerometers in both $x$ - and $y$-directions is estimated to

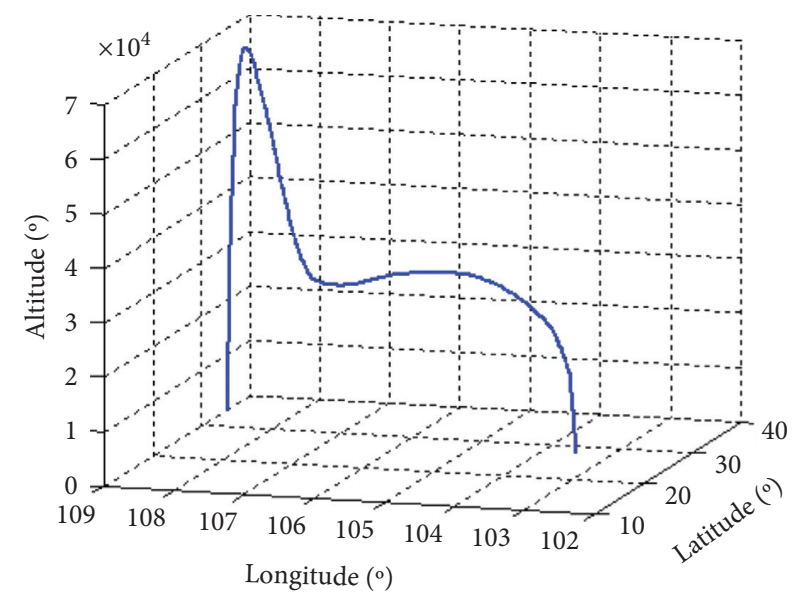

FIGURE 12: Flight trajectory of a hypersonic vehicle.

be $1.1 \mathrm{mg}$. Due to the small motion in the $z$-direction, the drift of accelerometers in this direction is estimated to be $0.7 \mathrm{mg}$. 

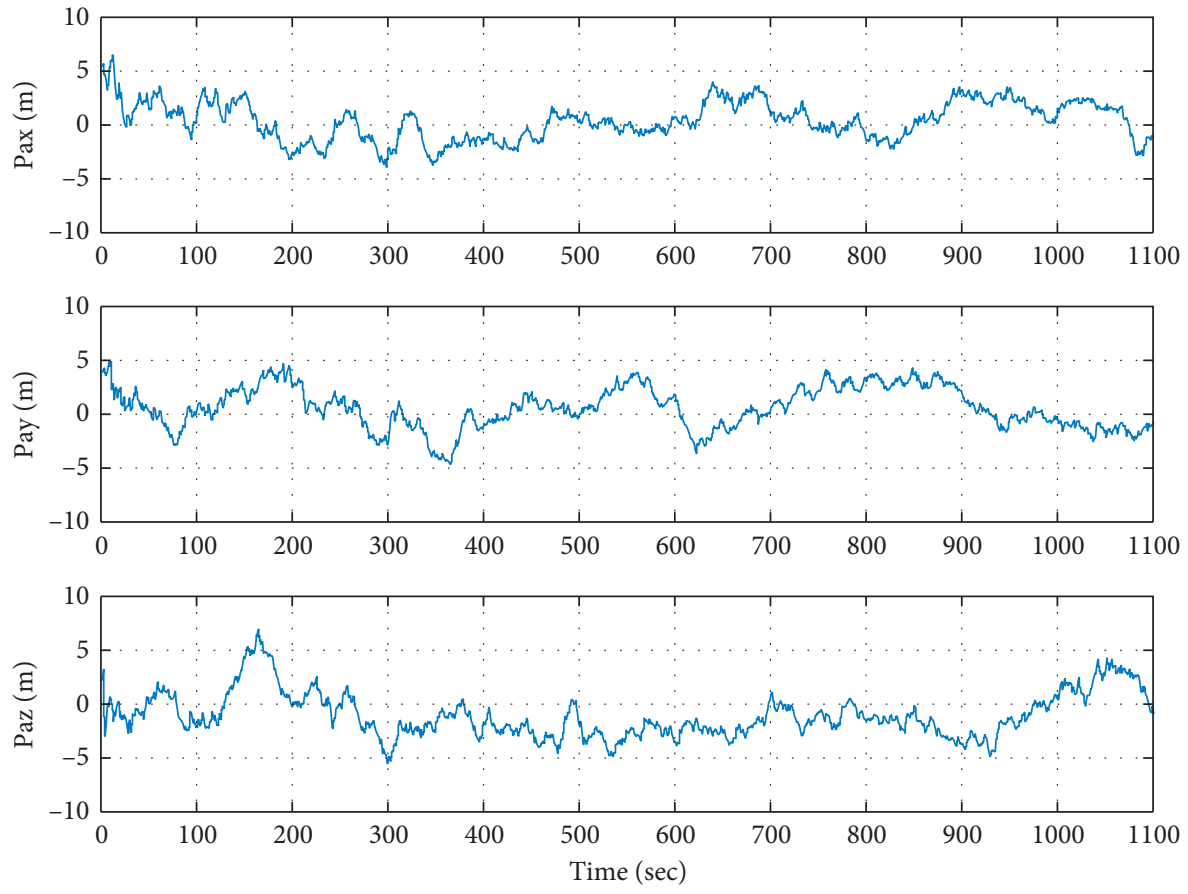

FIgURe 13: Position error in the LCI frame.
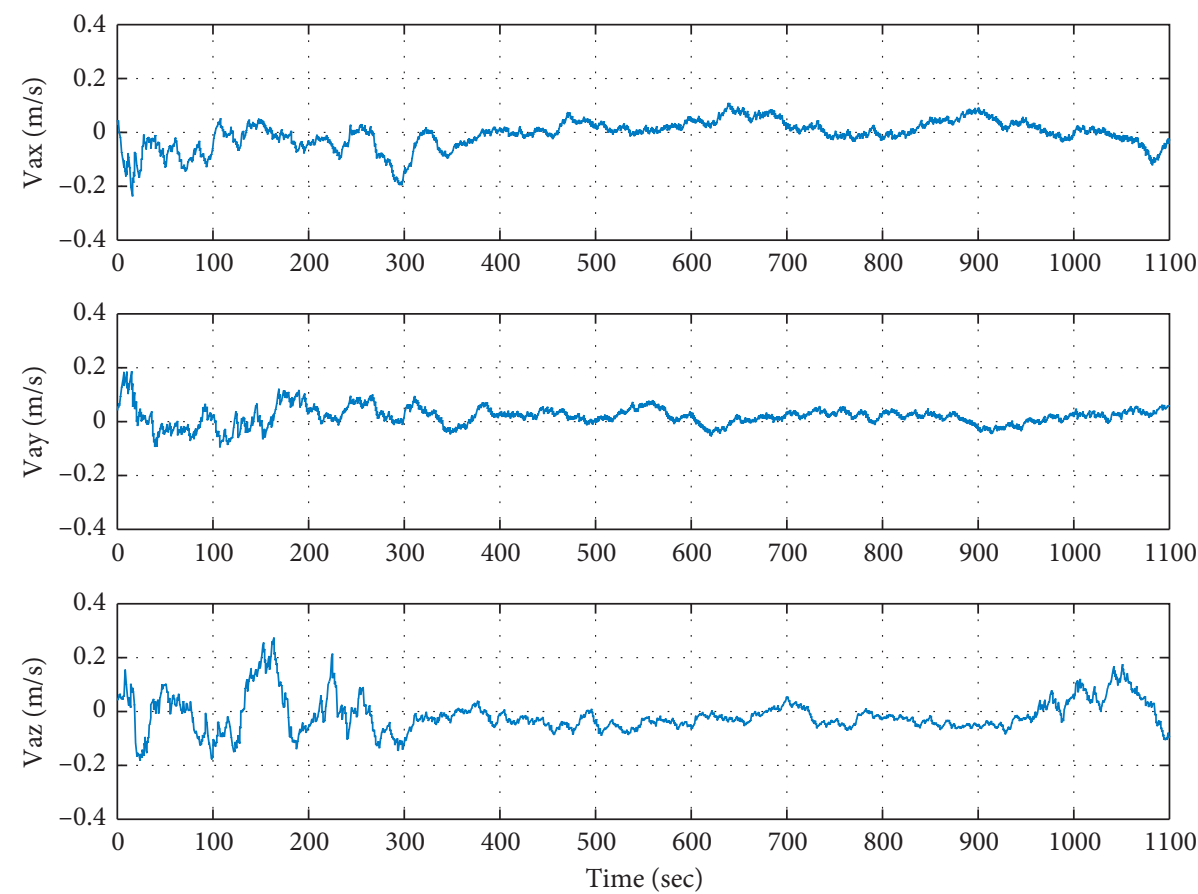

FIGURE 14: Velocity error in the LCI frame. 

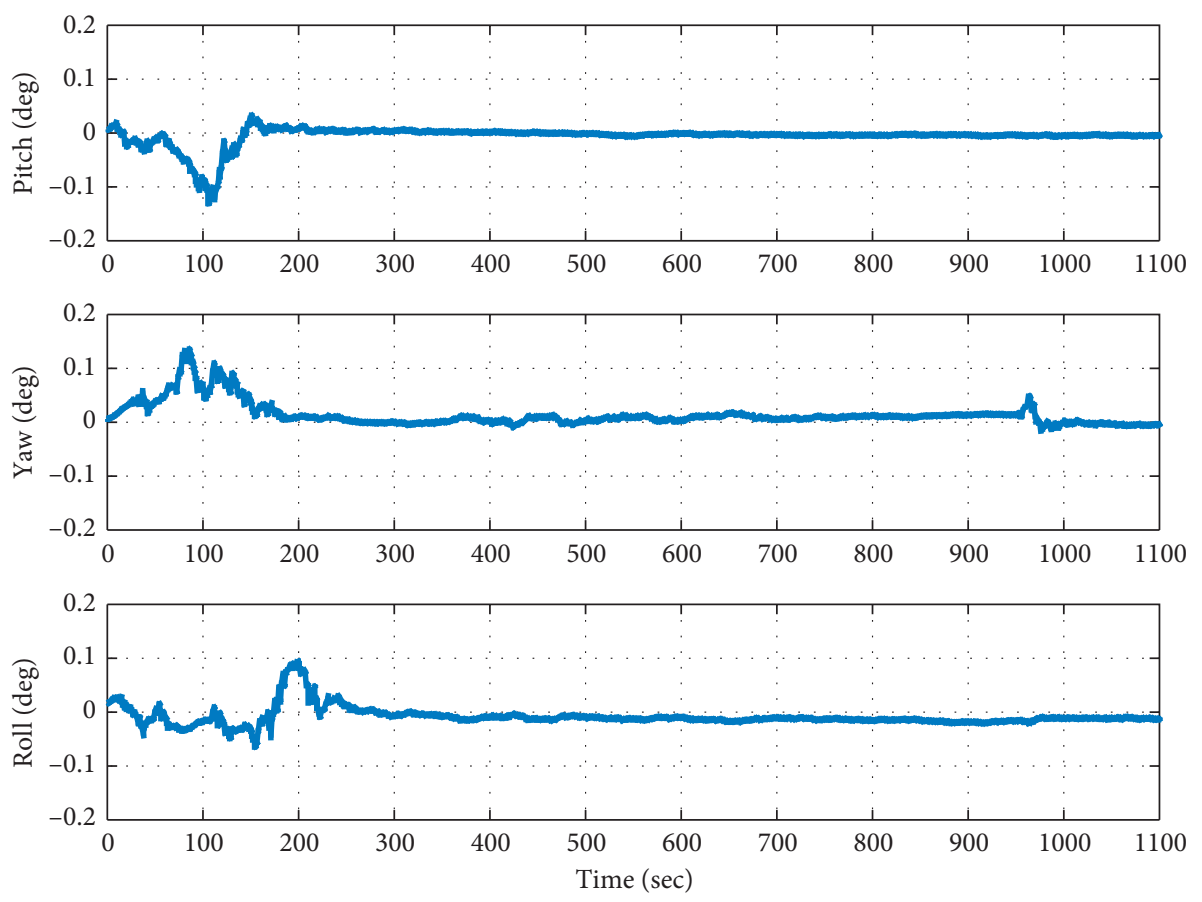

Figure 15: Attitude error in the LCI frame.
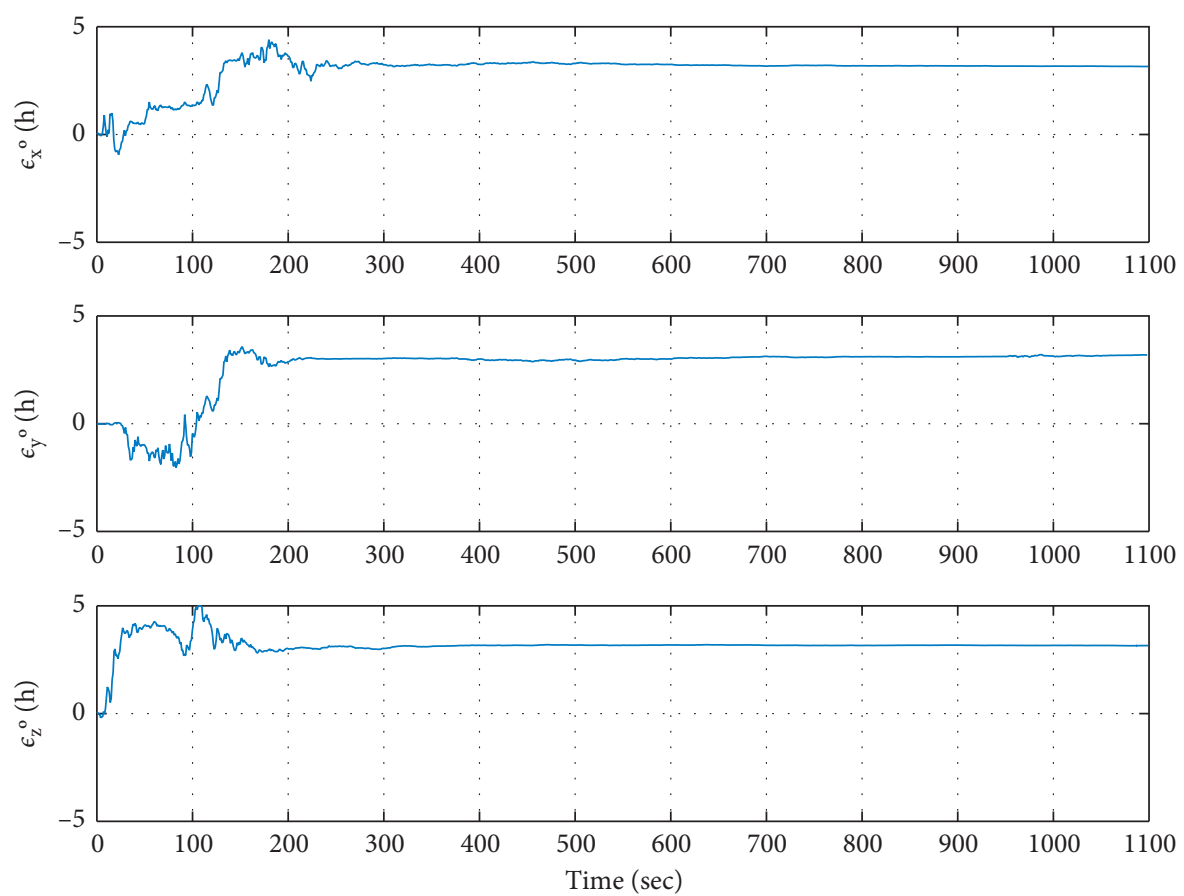

FIgURE 16: Gyroscope drift estimation in the LCI frame. 

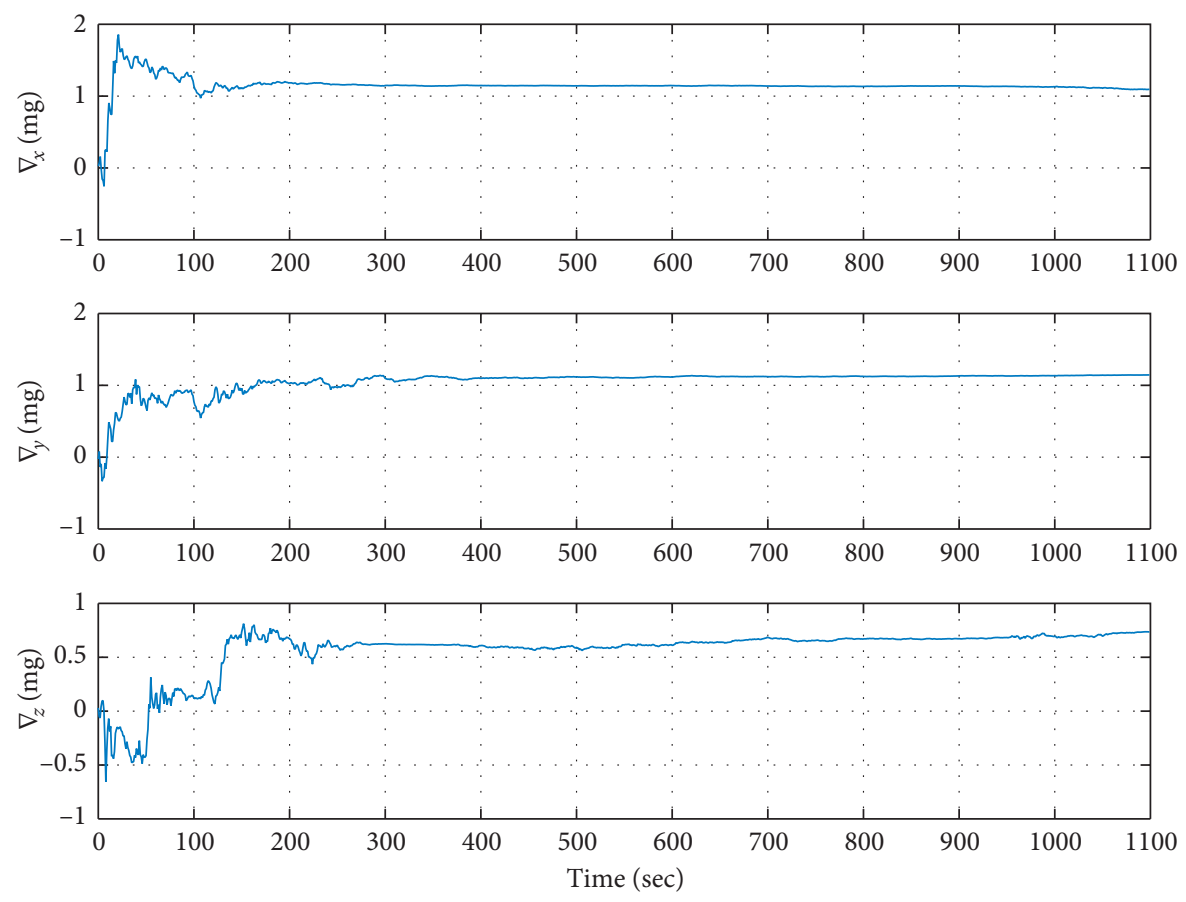

FIGURE 17: Accelerometer drift estimation in the LCI frame.

\section{Conclusions}

In this work, we considered the hypersonic boost-glide vehicles as the object, proposed the SINS mechanization and SINS numerical update algorithm in the LCI frame, and designed the SINS/BDS integrated algorithm in the LCI frame. In addition, we conducted vehicle field tests and hardware-in-the-loop simulations, and the accuracy of the simulation results was found to meet the navigation requirements. When the pitch angle is $90^{\circ}$, the attitude angle in the LCI frame is not singular, which meets the requirements for the vertical launch of the hypersonic boost-glide vehicles. Therefore, the proposed integrated navigation system is especially suitable for hypersonic boost-glide vehicles and other vertically launched aircrafts.

\section{Data Availability}

The data used in this article are not available for public disclosure.

\section{Conflicts of Interest}

The authors declare that they have no conflicts of interest.

\section{References}

[1] Y.r. Wu, P.f. Yang, and Y. l. Wu, "Stochastic control-oriented modeling of flexible air-breathing hypersonic vehicle," Mathematical Problems in Engineering, vol. 2018, pp. 1-12, Article ID 1648560, 2018.

[2] X. b. Zhang and Q. Zong, "Modeling and analysis of an airbreathing flexible hypersonic vehicle," Mathematical Problems in Engineering, vol. 2014, pp. 1-9, Article ID 264247, 2014.
[3] M. Duan and D. Zhou, "Finite-time composite guidance law with input constraint and dynamics compensation," Chinese Journal of Aeronautics, vol. 33, no. 2, pp. 664-671, 2019.

[4] K. Chen, J. Zhou, F.-Q. Shen, H.-Y. Sun, and H. Fan, "Hypersonic boost-glide vehicle strapdown inertial navigation system/global positioning system algorithm in a launchcentered earth-fixed frame," Aerospace Science and Technology, vol. 98, Article ID 105679, 2020.

[5] S. Stephen, "Real-time navigation algorithm for the SHEFEX2 hybrid navigation system experiment," in Proceedings of the AIAA Guidance, Navigation, and Control Conference Minnesota, Minnesota, MP, USA, August 2012.

[6] F. X. Mu, M. Ji, and Z. J. Zhang, "Hypersonic vehicle integrated navigation algorithm," Aeronautical Computing Technology, vol. 40, no. 5, pp. 55-58, 2010.

[7] M. Wei and K. P. Schwarz, "A strapdown inertial algorithm using an earth-fixed cartesian frame," Navigation, vol. 37, no. 2, pp. 153-167, 1990.

[8] Y.-j. Yu, J.-f. Xu, and Z. Xiong, "SINS/CNS nonlinear integrated navigation algorithm for hypersonic vehicle," Mathematical Problems in Engineering, vol. 2015, pp. 1-7, Article ID 903054, 2015.

[9] C. Bahm, E. Baumann, D. Bose, R. Beck, and B. Strovers, "The $\mathrm{X}-43 \mathrm{~A}$ hyper-X Mach 7 flight 2 guidance, navigation, and control overview and flight test results," in Proceedings of the AIAA/CIRA 13th International Space Planes and Hypersonic Systems and Technologies Conference, Capua, Italy, May 2005.

[10] X. C. Kang, G. J. He, and X. G. Li, "A SINS/BDS integrated navigation method based on classified weighted adaptive filtering," Mathematical Problems in Engineering, vol. 2019, pp. 1-6, Article ID 2158351, 2019.

[11] X. Y. Li, Research on Aerial Alignment Method of MissileBorne SINS/GPS Integrated Navigation system, North University of China, Taiyuan, China, 2019.

[12] H. Zhang, W. Zheng, and G. Tang, "Stellar/inertial integrated guidance for responsive launch vehicles," Aerospace Science and Technology, vol. 18, no. 1, pp. 35-41, 2012. 
[13] H. Zhao, Z. Xiong, L. Shi, F. Yu, and J. Liu, "A robust filtering algorithm for integrated navigation system of aerospace vehicle in launch inertial coordinate," Aerospace Science and Technology, vol. 58, pp. 629-640, 2016.

[14] J. Shi, C. Ouyang, Y. Huang, and W. Peng, "Assessment of BDS-3 global positioning service: ephemeris, SPP, PPP, RTK, and new signal," GPS Solut, vol. 24, p. 81, 2020.

[15] Q. Wang, S. Jin, L. Yuan, Y. Hu, J. Chen, and J. Guo, "Estimation and analysis of BDS-3 differential code biases from MGEX observations," Remote Sensing, vol. 12, p. 68, 2020.

[16] Y. Y. Ben, Q. Q. Gao, Q. Li, and X. Y. Liu, "A modified velocity updating algorithm for a strapdown INS," Measurement Science and Technology, vol. 31, no. 12, 2020.

[17] P. Jiang, Y. Zhang, Q. Hao, S. Fan, and D. Xu, "A Method converting cone into sculling algorithm for strapdown inertial navigation system," IEEE Access, vol. 7, pp. 140430-140437, 2019.

[18] P. G. Savage, "Strapdown inertial navigation integration algorithm design part 1: Attitude algorithms," Journal of Guidance, Control, and Dynamics, vol. 21, no. 1, pp. 19-28, 1998.

[19] P. G. Savage, "Strapdown inertial navigation integration algorithm design part 2: Velocity and position algorithms," Journal of Guidance, Control, and Dynamics, vol. 21, no. 2, pp. 208-221, 1998.

[20] K. Chen, F. Q. Shen, H. Y. Sun et al., "Hypersonic vehicle navigation algorithm in launch centered earth-fixed frame," Chinese Journal of Astronautics, vol. 40, no. 10, pp. 1212-1218, 2019, in Chinese.

[21] K. Chen, L. Y. Zhang, X. Wang, M. X. Liu, Y. F. Yu, and J. Yan, "Strapdown inertial navigation algorithm for hypersonic boost-glide vehicle," in Proceedings of the 21st AIAA International Space Planes and Hypersonics Technologies Conference, Xiamen, China, March 2017.

[22] K. Chen, F. Wei, Q. Zhang, Y. Yu, and J. Yan, "Trajectory generator of SINS on flight dynamics with application in hardware-in-the-loop simulation," Journal of Chinese Institute of Food Science and Technology, vol. 22, pp. 486-491, 2014.

[23] K. Chen, F. Shen, J. Zhou, and X. Wu, "Simulation platform for SINS/GPS integrated navigation system of hypersonic vehicles based on flight mechanics," Sensors, vol. 20, no. 18, p. $5418,2020$. 